\title{
SOME INTEGRAL EQUATIONS INVOLVING HYPERGEOMETRIC FUNCTIONS
}

\author{
by E. R. LOVE
}

(Received 16th June 1966)

Summary. An integral equation of the first kind, with kernel involving a hypergeometric function, is discussed. Conditions sufficient for uniqueness of solutions are given, then conditions necessary for existence of solutions. Conditions sufficient for existence of solutions, only a little stricter than the necessary conditions, are given; and with them two distinct forms of explicit solution. These two forms are associated at first with different ranges of the parameters, but their validity in the complementary ranges is also discussed. Before giving the existence theory a digression is made on a subsidiary integral equation.

Corresponding theorems for another integral equation resembling the main one are deduced from some of the previous theorems. Two more equations of similar form, less closely related, will be considered in another paper. Special cases of some of these four integral equations have been considered recently by Erdélyi, Higgins, Wimp and others.

\section{Introduction}

Since $1960 \mathrm{Ta} \mathrm{Li} \mathrm{(1),} \mathrm{Buschman} \mathrm{(2)} \mathrm{and} \mathrm{Higgins} \mathrm{(3)} \mathrm{have} \mathrm{solved} \mathrm{integral}$ equations which are special cases of

$$
\int_{x}^{1}\left(t^{2}-x^{2}\right)^{-\frac{1}{2} \mu} P_{v}^{\mu}\left(\frac{t}{x}\right) f(t) d t=g(x) \text { for } \alpha \leqq x \leqq 1,
$$

where $\alpha>0$; the values of $\mu$ and $\nu$ being quite specialised. Srivastava (4) considered this equation with somewhat less specialised values of $\mu$ and $v$.

More generally, Erdélyi (5) solved the similar equation

$$
\int_{a}^{x}\left(x^{2}-t^{2}\right)^{-\frac{1}{2} \mu} P_{v}^{\mu}\left(\frac{x}{t}\right) f(t) d t=g(x) \text { for } \alpha \leqq x \leqq \beta
$$

with $\alpha>0, \mu$ and $v$ being unrestricted except for $\operatorname{Re} \mu<1$.

Higgins (6) and Wimp (7) solved some integral equations involving the hypergeometric function $F(a, b, c, z)$. One of these is

$$
\int_{x}^{1} \frac{(t-x)^{c-1}}{\Gamma(c)} F\left(a, b, c, 1-\frac{t}{x}\right) f(t) d t=g(x) \text { for } \alpha \leqq x \leqq 1 ;
$$


the other differs only in having $x / t$ in place of $t / x$. Equation (1.3) includes (1.1), reducing to it by simple transformations when $c-a-b=\frac{1}{2}$.

$I$ discuss here the integral equation

$$
\int_{0}^{x} \frac{(x-t)^{c-1}}{\Gamma(c)} F\left(a, b, c, 1-\frac{x}{t}\right) f(t) d t={ }^{\circ} g(x) \text { for } 0<x<d,
$$

where $0<d \leqq \infty$ and $={ }^{\circ}$ means " equals almost everywhere". This equation reduces to (1.2) when the parameters $a, b, c$ are specially related and when $g$ vanishes in a neighbourhood of 0 (see Theorem 4, Corollary 2). But with 0 in the supports of $f$ and $g,(1.4)$ has some extra complications associated with the singular points 1 and $\infty$ of the hypergeometric function.

The functions $f$ and $g$ are representatives of classes of equivalent functions, and Lebesgue integrals only are used; this makes the hypothesis $\operatorname{Re} c>0$ requisite throughout. Professor Erdélyi tells me that he has considered the Legendre case (1.2) with finite part integrals and $\operatorname{Re} \mu>1$, corresponding to $\operatorname{Re} c<0$. I take this opportunity of thanking Professor Erdélyi for his interest in this work, and for several stimulating questions which have led to substantial improvements. His paper (5) and Srivastava's (8) first interested me in the subject.

In $\S 10$, the last section of the paper, I discuss the similar equation

$$
\int_{0}^{x} \frac{(x-t)^{c-1}}{\Gamma(c)} F\left(a, b, c, 1-\frac{t}{x}\right) f(t) d t={ }^{\circ} g(x) \text { for } 0<x<d,
$$

deducing theorems on uniqueness and existence of solutions, and explicit solutions, from some of the theorems already obtained for (1.4). In another paper $\dagger$ I hope to consider two more hypergeometric equations of the same form as (1.4) and (1.5), with range of integration $(x, \infty)$ instead of $(0, x)$.

In $\S 5$ a digression is made to obtain existence theorems for solutions of

$$
\int_{0}^{x} \frac{(x-t)^{b-1}}{\Gamma(b)} t^{a} f(t) d t={ }^{\circ} x^{a} \int_{0}^{x} \frac{(x-t)^{b-1}}{\Gamma(b)} g(t) d t
$$

supposing either $f$ or $g$ to be unknown. These theorems play an essential part in the treatment of (1.4); and convenient explicit solutions of (1.6), in terms of hypergeometric functions, are obtained incidentally.

Apart from the sections mentioned in the two preceding paragraphs the paper is concerned entirely with (1.4). The contents of the remaining sections are outlined below.

Preliminary theorems on convergence of the left side of (1.4) and on fractional integration of it occupy $\S 2$ and $\S 3$. Theorem 1 in particular supplies the justification for the inversions of order of integration. Remarks on its hypothesis show that a little relaxation is possible, but a counter-example limiting this possibility is also given.

$\dagger$ To appear in Proc. Camb. Phil. Soc. 
Theorem 4 on uniqueness of solutions of (1.4) occupies $\S 4$. A corollary shows that the present theory is applicable, under suitable conditions, to a truncated form of (1.4) with lower terminal positive, and in particular to (1.2).

The uniqueness theorem is relevant in another respect as well. At least six different formulae express solutions of (1.4) under various sets of conditions, and these sets are not disjoint. Three of these solution formulae are

$$
\begin{aligned}
& f(x)=x^{-a} I^{-b} x^{a} I^{b-c} g(x), \\
& f(x)=x^{-b} I^{b-c} x^{c-a} I^{-b} x^{a+b-c} g(x), \\
& f(x)=x^{-a} \frac{d^{m}}{d x^{m}}\left\{x^{a} \int_{0}^{x} \frac{(x-t)^{m-c-1}}{\Gamma(m-c)} F\left(-a, m-b, m-c, 1-\frac{t}{x}\right) g(t) d t\right\},
\end{aligned}
$$

where $I^{\lambda}$ is the fractional integration operator used throughout (see $\S 3$ ) and $m$ is any integer exceeding $\max (\operatorname{Re} b, \operatorname{Re} c, \operatorname{Re}(c-b))$. The other three solution formulae mentioned are obtained from these by interchanging $a$ and $b$.

Necessary conditions for existence of solutions of (1.4) are given) in $\S 6$. Here, and throughout, "solution" means "solution in $Q_{q}$ "; $Q_{q}$ being a class of functions which are locally integrable in $(0, d)$ and have special behaviour at 0 . The definition of $Q_{q}$ is given in $\S 5$, just before Theorem 5 .

Sufficient conditions for existence of solutions of (1.4) are given in $\S 7$, and also explicit solutions. The sufficient conditions are only slightly different from the necessary conditions of $\S 6$, as can be seen by a comparison of Theorems 7 and 10. The explicit solutions are (1.7) and (1.8), associated with specified domains of the parameters.

The theorems in $\S 8$ show that (1.7) and (1.8) remain solutions of (1.4) outside the domain of $b$ with which they were associated in $\S 7$, provided that an extra restriction is placed on the right-hand side $g$. The extra restriction is a higher degree of differentiability, and then $f$ is correspondingly more highly differentiable.

In $\S 9$ several variants of Theorems 7, 8, 9 and 10 are given. These all refer to the degree of differentiability of $g$ that is necessary (in the versions of Theorem 7) and sufficient (in the others) for existence of solutions. They depend on corollaries of Theorems 5 and 6 .

\section{Existence of the integrals}

The following theorem resembles the standard theorem on existence and integrability of a fractional integral (see (3.1)), that if $\operatorname{Re} k>0$ and $f$ is integrable on $(0, l)$, then $I^{k} f$ is integrable on $(0, l)$; where

$$
I^{k} f(x)=\int_{0}^{x} \frac{(x-t)^{k-1}}{\Gamma(k)} f(t) d t .
$$

Our theorem is almost, but not quite, a generalisation of this. The relationship between them will be made clearer in the remarks after the proof. 
Theorem 1. If $\operatorname{Re} k>0, q \leqq \operatorname{Re}(h+k), q<\min (\operatorname{Re} a, \operatorname{Re} b), c \neq 0,-1$, $-2, \ldots$ and $x^{q} f(x)$ is integrable on a finite interval $(0, l)$, then

$$
x^{h} \int_{0}^{x} \frac{(x-t)^{k-1}}{\Gamma(k)} F\left(a, b, c, 1-\frac{x}{t}\right) f(t) d t
$$

exists almost everywhere in $(0, l)$ and is integrable on it. The same is true of the function obtained by replacing the integrand by its modulus.

The integrand in (2.1) is a measurable function of $t$ since $t^{-q} \cdot t^{q} f(t)$ is measurable; and it is also a measurable function of $(x, t)$, so that

$$
\left|x^{h}\right| \int_{0}^{x}\left|\frac{(x-t)^{k-1}}{\Gamma(k)} F\left(a, b, c, 1-\frac{x}{t}\right) f(t)\right| d t
$$

is a measurable function of $x$ on $(0, l)$, finite or infinite, by Fubini's theorem. We prove that $(2.2)$ is integrable on $(0, l)$, and from this the other statements made are immediate corollaries.

It is thus enough to prove the finiteness of

$$
\begin{aligned}
\int_{0}^{t} \mid & x^{h}\left|d x \int_{0}^{x}\right|(x-t)^{k-1} F\left(a, b, c, 1-\frac{x}{t}\right) f(t) \mid d t \\
& =\int_{0}^{t}|f(t)| d t \int_{t}^{t}\left|x^{h}(x-t)^{k-1} F\left(a, b, c, 1-\frac{x}{t}\right)\right| d x \\
& =\int_{0}^{l}\left|f(t) t^{h+k}\right| d t \int_{0}^{l / t-1}\left|(1+s)^{h} s^{k-1} F(a, b, c,-s)\right| d s \quad[x=t(1+s)] \\
& \leqq \\
& \int_{0}^{t}\left|f(t) t^{h+k}\right| d t \int_{0}^{3}\left|(1+s)^{h} s^{k-1} F(a, b, c,-s)\right| d s \\
& \quad+\int_{0}^{l / 4}\left|f(t) t^{h+k}\right| d t \int_{3}^{l / t-1}\left|(1+s)^{h} s^{k-1} F(a, b, c,-s)\right| d s .
\end{aligned}
$$

We prove that (2.3) and (2.4) are finite. We write $\alpha, \beta, \gamma, \theta, \kappa$ respectively for $\operatorname{Re} a, \operatorname{Re} b, \operatorname{Re} c, \operatorname{Re} h, \operatorname{Re} k$, and suppose $\alpha \leqq \beta$ as we may because of the symmetry of the hypergeometric function.

The two integrals into which (2.3) is factorised are finite. For

$$
\left|f(t) t^{h+k}\right|=|f(t)| t^{\theta+\kappa}=t^{q}|f(t)| t^{\theta+\kappa-q} \leqq\left|t^{q} f(t)\right| l^{\theta+\kappa-q}
$$

since $q \leqq \theta+\kappa$; thus the $t$-integral is finite since $t^{q} f(t)$ is integrable. And all factors in the $s$-integrand are continuous in $0 \leqq s \leqq 3$, and so bounded, except $\left|s^{k-1}\right|=s^{\kappa-1}$ which is integrable since $\kappa>0$.

It remains to prove (2.4) finite. Choose $\mu$ such that

$$
q<\mu<\alpha \text { and } \mu \neq \theta+\kappa .
$$

Since $a$ and $b$ are the exponents at $\infty$ of the hypergeometric equation satisfied by $F(a, b, c,-s)$, and this function is continuous in $s \geqq 3$,

$$
|F(a, b, c,-s)| \leqq A s^{-\alpha}+B s^{-\beta} \log s \leqq C s^{-\mu}
$$


in $s \geqq 3$, where $A, B, C$ are independent of $s$. So (2.4) is at most

$$
\int_{0}^{1 / 4}|f(t)| t^{\theta+\kappa} d t \int_{3}^{1 / t-1}(1+s)^{\theta} s^{\kappa-1} C s^{-\mu} d s .
$$

To estimate the inner integral in (2.8) we have, if $\kappa-\mu-1 \leqq 0$,

$$
s^{x-\mu-1} \leqq 2^{-\kappa+\mu+1}(1+s)^{x-\mu-1}
$$

in the range of integration, since $s>\frac{1}{2} s+\frac{1}{2}$ if $s>1$. The same inequality without the power of 2 holds if $\kappa-\mu-1>0$. So if we write $C^{\prime}$ for $C \max \left(2^{-x+\mu+1}, 1\right)$, the inner integral in (2.8) is at most

$$
C^{\prime} \int_{3}^{l / t-1}(1+s)^{\theta+\kappa-\mu-1} d s=\frac{C^{\prime}}{\theta+\kappa-\mu}\left\{\left(\frac{l}{t}\right)^{\theta+\kappa-\mu}-4^{\theta+\kappa-\mu}\right\},
$$

remembering that $\theta+\kappa-\mu \neq 0$, by (2.6).

If $\theta+\kappa-\mu>0,(2.8)$ is at most

$$
\frac{C^{\prime}}{\theta+\kappa-\mu} \int_{0}^{1 / 4}|f(t)| t^{\theta+\kappa}\left(\frac{l}{t}\right)^{\theta+\kappa-\mu} d t=\frac{C^{\prime} l^{\theta+\kappa-\mu}}{\theta+\kappa-\mu} \int_{0}^{l / 4}|f(t)| t^{\mu} d t,
$$

and this is convergent since $\mu>q$ by (2.6) and so $t^{\mu} \leqq(l / 4)^{\mu-q_{t} q}$.

If $\theta+\kappa-\mu<0,(2.8)$ is at most

$$
\frac{C^{\prime} 4^{\theta+\kappa-\mu}}{\mu-\theta-\kappa} \int_{0}^{1 / 4}|f(t)| t^{\theta+\kappa} d t
$$

and this is convergent since $\theta+\kappa \geqq q$ and (2.5) applies.

These two estimates show that (2.8), and therefore (2.4), is always finite as required.

Remark 1. Theorem 1 remains true if the two inequalities restricting $q$ are replaced by: $q<\operatorname{Re}(h+k), q \leqq \min (\operatorname{Re} a, \operatorname{Re} b)$, and $a \neq b$.

The preceding proof fails only if $q=\min (\operatorname{Re} a, \operatorname{Re} b)$, and in that case it is still applicable until (2.6) is reached. Supposing this case, that is, $q=\alpha \leqq \beta, \mu$ cannot be chosen to satisfy (2.6). But (2.7) holds with $\mu=\alpha$ : obviously if $\alpha<\beta$, but also if $\alpha=\beta$. For the logarithm can be omitted if $b-a$ is not an integer, and this is necessarily so as $\alpha=\beta$ and $a \neq b$. Thus (2.8) stands with $\mu=\alpha$.

With our present data, then, $\mu=\alpha=q<\theta+\kappa$. So $\theta+\kappa-\mu>0$, whence (2.9) and (2.10) also stand. The final integral in (2.10) is convergent since $\mu=q$, and this completes the proof since the case $\theta+\kappa-\mu<0$ cannot now occur.

Remark 2. Theorem 1 becomes false if the two inequalities restricting $q$ are replaced by

$$
q \leqq \min (\operatorname{Re}(h+k), \operatorname{Re} a, \operatorname{Re} b) ;
$$

and it makes no difference whether $a$ and $b$ are equal or not. This is shown by the case

$$
h=0, k=1, q=1, a=1, b=c \geqq 1, f(t)=\{t \log (3 l / t)\}^{-2} .
$$


For $t^{q} f(t)$ is now integrable on $(0, l)$, but (2.1) is not, being equal to

$$
\int_{0}^{x}\left(\frac{x}{t}\right)^{-1} f(t) d t=\frac{1}{x} \int_{0}^{x} \frac{d t}{t\{\log (3 l / t)\}^{2}}=\frac{1}{x \log (3 l / x)}
$$

Remark 3. The alternative version of Theorem 1 given in Remark 1 includes the standard theorem on integrability of a fractional integral mentioned at the beginning of $\S 2$, but the original version does not. This is apparent by putting $h=0, q=0, a=0, b=c=1$.

Remark 4. The original version of Theorem 1 includes the following theorem on integrability of fractional integrals. If $\operatorname{Re} k>0, q<\operatorname{Re} a$ and $x^{q} f(x)$ is integrable on $(0, l)$ then so is

$$
x^{q-a-k} \int_{0}^{x} \frac{(x-t)^{k-1}}{\Gamma(k)} t^{a} f(t) d t .
$$

This is the special case $h=q-k, b=c=a+|\operatorname{Re} a|+1$ of Theorem 1 . The particular case $q=0$ of this is also a particular case of a theorem of Kober (9).

\section{Other preliminaries}

Integrability. We consider complex-valued functions on a real interval $[0, d)$, where $d \leqq \infty$. Often they are locally integrable on $[0, d)$, that is, $L$ integrable on $(0, l)$ for each $l<d$. Functions whose values agree almost everywhere in $(0, d)$ are called equivalent and regarded as indistinguishable; and functions need only be defined almost everywhere in $(0, d)$.

Fractional integrals. For complex numbers $c$ with $\operatorname{Re} c>0$, and functions $f$ locally integrable on $[0, d), I^{c} f$ is the function whose values are

$$
I^{c} f(x)=\int_{0}^{x} \frac{(x-t)^{c-1}}{\Gamma(c)} f(t) d t .
$$

It is a standard theorem that this defines $I^{c} f(x)$ for almost all $x$ and that $I^{c} f$ is locally integrable on $[0, d)$; this is the theorem mentioned in $\S 2$, at the beginning and again in Remark 3 .

It is also well known that if $\operatorname{Re} a>0, \operatorname{Re} b>0$ and $f$ is locally integrable then

$$
I^{b} I^{a} f=I^{a+b} f \text {. }
$$

Fractional derivatives. If $\operatorname{Re} c>0, I^{-c} f$ is defined as a locally integrable function $\phi$ such that $I^{c} \phi=f$. Evidently $f$ must be locally integrable; but this does not ensure existence of $I^{-c} f$, as the case $c=1$ shows.

Uniqueness of $I^{-c} f$ is ensured by the following well-known theorem: If $\operatorname{Re} c>0$ and $\phi$ is locally integrable on $[0, d)$, then

$$
I^{c} \phi(x)={ }^{\circ} 0 \text { implies that } \phi(x)={ }^{\circ} 0 \text {. }
$$

It follows from (3.2) that if $\operatorname{Re} a>0, \operatorname{Re} b>0$ and $I^{-a-b} f$ exists, then $I^{-a} I^{-b} f$ exists and

$$
I^{-a} I^{-b} f=I^{-a-b} f
$$


It should perhaps be added that (3.4), like (3.2), means that the functions equated are defined almost everywhere and are equivalent.

Hypergeometric integrals. For complex numbers $a, b, c$ with $\operatorname{Re} c>0$, and suitable functions $f$, we define $H(a, b, c) f$ to be the function whose values are

$$
H(a, b, c) f(x)=\int_{0}^{x} \frac{(x-t)^{c-1}}{\Gamma(c)} F\left(a, b, c, 1-\frac{x}{t}\right) f(t) d t .
$$

Theorem 1 shows that $H(a, b, c) f$ is locally integrable on $[0, d)$ if $x^{a} f(x)$ is, where $q \leqq \operatorname{Re} c$ and $q<\min (\operatorname{Re} a, \operatorname{Re} b)$.

Lemma 1. If $\operatorname{Re} \lambda>0, \operatorname{Re} c>0$ and $z$ is in the complex plane cut along $z \geqq 1$, then we have "Bateman's integral"

$$
\int_{0}^{1} \frac{(1-u)^{\lambda-1}}{\Gamma(\lambda)} \frac{u^{c-1}}{\Gamma(c)} F(a, b, c, z u) d u=\frac{1}{\Gamma(c+\lambda)} F(a, b, c+\lambda, z) .
$$

In particular, if $\operatorname{Re} \lambda>0, \operatorname{Re} c>0$ and $0<t<x$, then

$$
\begin{aligned}
\int_{t}^{x} \frac{(x-s)^{\lambda-1}}{\Gamma(\lambda)} \frac{(s-t)^{c-1}}{\Gamma(c)} F\left(a, b, c, 1-\frac{s}{t}\right) d s & \\
& =\frac{(x-t)^{c+\lambda-1}}{\Gamma(c+\lambda)} F\left(a, b, c+\lambda, 1-\frac{x}{t}\right) \\
\int_{t}^{x} \frac{(x-s)^{c-1}}{\Gamma(c)} \frac{(s-t)^{\lambda-1}}{\Gamma(\lambda)} F(a, b, c, & \left.1-\frac{s}{x}\right) d s \\
& =\frac{(x-t)^{c+\lambda-1}}{\Gamma(c+\lambda)} F\left(a, b, c+\lambda, 1-\frac{t}{x}\right) .
\end{aligned}
$$

What I have called Bateman's integral (3.6) is, after change of notation, [(10), 2.4(2)]. Formulae (3.7) and (3.8) are obtained from it quite directly by changes of scale; in fact, by the substitutions

$$
\begin{aligned}
& z=\frac{t-x}{t} \text { and } u=\frac{s-t}{x-t} \text { for (3.7), } \\
& z=\frac{x-t}{x} \text { and } u=\frac{x-s}{x-t} \text { for (3.8). }
\end{aligned}
$$

It would be incorrect to say that (3.8) is got from (3.7) by interchanging $x$ and $t$, because both formulae presuppose that $0<t<x$; this restriction becomes significant when index laws are used with the principal value powers occurring.

Theorem 2. If $\operatorname{Re} \lambda>0, \operatorname{Re} c>0, q \leqq \operatorname{Re} c, q<\min (\operatorname{Re} a, \operatorname{Re} b)$, and $x^{q} f(x)$ is locally integrable on $[0, d)$, then $H(a, b, c) f$ is also locally integrable and

$$
I^{\lambda} H(a, b, c) f=H(a, b, c+\lambda) f
$$


that is to say, for almost all $x$ in $[0, d)$,

$$
\begin{aligned}
\int_{0}^{x} \frac{(x-s)^{\lambda-1}}{\Gamma(\lambda)} d s \int_{0}^{s} \frac{(s-t)^{c-1}}{\Gamma(c)} F\left(a, b, c, 1-\frac{s}{t}\right) f(t) d t & \\
& =\int_{0}^{x} \frac{(x-t)^{c+\lambda-1}}{\Gamma(c+\lambda)} F\left(a, b, c+\lambda, 1-\frac{x}{t}\right) f(t) d t .
\end{aligned}
$$

Fixing $l$ such that $0<l<d$, we have only to prove this equation for almost all $x$ in $(0, l)$, and integrability of $H(a, b, c) f$ on $(0, l)$. The integrability follows from Theorem 1 with $h=0$ and $k=c$. And the double integral is absolutely convergent for almost all $x$ in $(0, l)$, because

$$
\int_{0}^{x}\left|\frac{(x-s)^{\lambda-1}}{\Gamma(\lambda)}\right|\left\{\int_{0}^{s}\left|\frac{(s-t)^{c-1}}{\Gamma(c)} F\left(a, b, c, 1-\frac{s}{t}\right) f(t)\right| d t\right\} d s
$$

is a convolution of integrable functions, $x^{R e \lambda-1} /|\Gamma(\lambda)|$ and the function (2.2) with $h=0$ and $k=c$, which is also integrable by Theorem 1 . The convolution is finite almost everywhere, so the double integral is absolutely convergent almost everywhere.

Inverting the order of integration, then, the left side of (3.9) becomes

$$
\int_{0}^{x} f(t) d t \int_{t}^{x} \frac{(x-s)^{\lambda-1}}{\Gamma(\lambda)} \frac{(s-t)^{c-1}}{\Gamma(c)} F\left(a, b, c, 1-\frac{s}{t}\right) d s
$$

for almost all $x$, and this is equal to the right side by Lemma 1 (3.7), as required.

Theorem 3. If $\operatorname{Re} \lambda>0, \operatorname{Re} c>0, q \leqq \operatorname{Re}(c+\lambda), q<\min (\operatorname{Re} a, \operatorname{Re}(b+\lambda))$, and $x^{a} f(x)$ is locally integrable on $[0, d)$, then

$$
H(a, b, c) x^{-a} I^{\lambda} x^{a} f(x)={ }^{\circ} H(a, b+\lambda, c+\lambda) f(x) ;
$$

that is to say, for almost all $x$ in $[0, d)$,

$$
\begin{aligned}
\int_{0}^{x} \frac{(x-s)^{c-1}}{\Gamma(c)} F\left(a, b, c, 1-\frac{x}{s}\right) & s^{-a} d s \int_{0}^{s} \frac{(s-t)^{\lambda-1}}{\Gamma(\lambda)} t^{a} f(t) d t \\
& =\int_{0}^{x} \frac{(x-t)^{c+\lambda-1}}{\Gamma(c+\lambda)} F\left(a, b+\lambda, c+\lambda, 1-\frac{x}{t}\right) f(t) d t .
\end{aligned}
$$

Fixing $l$ such that $0<l<d$, we have only to prove this equation for almost all $x$ in $(0, l)$. To prove the double integral absolutely convergent for almost all $x$ in $(0, l)$ we write

$$
\begin{aligned}
\phi(s) & =\left|s^{-a}\right| \int_{0}^{s}\left|\frac{(s-t)^{\lambda-1}}{\Gamma(\lambda)} t^{a} f(t)\right| d t, \\
& =\int_{0}^{s}\left|\frac{(s-t)^{\lambda-1}}{\Gamma(\lambda)} F\left(a, c+\lambda+1, c+\lambda+1,1-\frac{s}{t}\right) f(t)\right| d t .
\end{aligned}
$$

Now $s^{q-\lambda} \phi(s)$ is integrable on $(0, l)$, by Theorem 1 with $h=q-\lambda$ and $k=\lambda$. Hence $s^{q^{\prime}} \phi(s)$ is also, where $q^{\prime}=q-$ re $\lambda$. So by another application of 
Theorem 1, with $h=0, k=c, f$ and $q$ replaced by $\phi$ and $q^{\prime}$,

$$
\int_{0}^{x}\left|\frac{(x-s)^{c-1}}{\Gamma(c)} F\left(a, b, c, 1-\frac{x}{s}\right) \phi(s)\right| d s
$$

exists almost everywhere in $(0, l)$. So the double integral in $(3.10)$ is absolutely convergent for almost all $x$ in $(0, l)$, and we may invert the order of integration.

The double integral is thus, for almost all $x$, equal to

$$
\int_{0}^{x} t^{a} f(t) d t \int_{t}^{x} \frac{(x-s)^{c-1}}{\Gamma(c)} \frac{(s-t)^{\lambda-1}}{\Gamma(\lambda)} F\left(a, b, c, 1-\frac{x}{s}\right) s^{-a} d s .
$$

Applying Kummer's relation [(10), 2.9(3)] to the hypergeometric function, the inner integral of (3.11) is equal to

$$
\begin{aligned}
\int_{t}^{x} \frac{(x-s)^{c-1}}{\Gamma(c)} \frac{(s-t)^{\lambda-1}}{\Gamma(\lambda)} F( & \left.a, c-b, c, 1-\frac{s}{x}\right) x^{-a} d s \\
& =\frac{(x-t)^{c+\lambda-1}}{\Gamma(c+\lambda)} F\left(a, c-b, c+\lambda, 1-\frac{t}{x}\right) x^{-a} \\
& =\frac{(x-t)^{c+\lambda-1}}{\Gamma(c+\lambda)} F\left(a, b+\lambda, c+\lambda, 1-\frac{x}{t}\right) t^{-a}
\end{aligned}
$$

where we have evaluated the integral by Lemma 1 (3.8), giving (3.12), and then used Kummer's relation again. Substituting the resulting expression for the inner integral in (3.11), the proof is complete.

Remark. There is a formal similarity between Theorems 2 and 3, although not between the proofs given. Noticing that the restrictions on $q$ used in Theorem 3 are exactly those which Theorem 1 would prescribe as sufficient, and nearly necessary, for existence of the integral on the right of (3.10), one might ask whether it should be likewise in Theorem 2; that is, whether $q \leqq \operatorname{Re} c$ in Theorem 2 ought to be replaced by $q \leqq \operatorname{Re}(c+\lambda)$. The following example shows that $q \leqq \operatorname{Re} c$ in Theorem 2 cannot be improved on.

Suppose that $a, b, c$ are real, $0<c<q<\min (a, b)$, and $b=c+1$. Let $\delta=\frac{1}{2}(q-c)>0$ and $f(x)=x^{-c-\delta-1}$. Then $x^{q} f(x)=\dot{x}^{\delta-1}$ is locally integrable on $[0, d)$, and all hypotheses of Theorem 2 are fulfilled except $q \leqq \operatorname{Re} c$. We show that the left side of (3.9) is divergent.

Since

$$
\begin{gathered}
F\left(a, c+1, c, 1-\frac{s}{t}\right)=\left(1-\frac{a}{c}\right)\left(\frac{s}{t}\right)^{-a}+\frac{a}{c}\left(\frac{s}{t}\right)^{-a-1} \\
\int_{0}^{s} \frac{(s-t)^{c-1}}{\Gamma(c)} F\left(a, b, c, 1-\frac{s}{t}\right) f(t) d t \\
=\left(1-\frac{a}{c}\right) s^{-a} \int_{0}^{s} \frac{(s-t)^{c-1}}{\Gamma(c)} t^{a-c-\delta-1} d t+\frac{a}{c} s^{-a-1} \int_{0}^{s} \frac{(s-t)^{c-1}}{\Gamma(c)} t^{a-c-\delta} d t
\end{gathered}
$$


these integrals being convergent since $a-c-\delta>q-c-\delta=\delta>0$. This is

$$
\begin{aligned}
& =\left(1-\frac{a}{c}\right) \frac{\Gamma(a-c-\delta)}{\Gamma(a-\delta)} s^{-\delta-1}+\frac{a}{c} \frac{\Gamma(a-c-\delta+1)}{\Gamma(a-\delta+1)} s^{-\delta-1} \\
& =-\frac{\delta}{a-\delta} \frac{\Gamma(a-c-\delta)}{\Gamma(a-\delta)} s^{-\delta-1}
\end{aligned}
$$

The double integral on the left of (3.9) is consequently divergent.

\section{Uniqueness of solutions of (1.4)}

Theorem 4. $\dagger$ If $\operatorname{Re} c>0, q \leqq \min (\operatorname{Re} c, \operatorname{Re}(a+b)), q<\min (\operatorname{Re} a, \operatorname{Re} b)$, $x^{q} f(x)$ is locally integrable on $[0, d)$ and

$$
\int_{0}^{x} \frac{(x-t)^{c-1}}{\Gamma(c)} F\left(a, b, c, 1-\frac{x}{t}\right) f(t) d t={ }^{\circ} 0
$$

then $f(x)={ }^{\circ} 0$.

(i) Suppose first that $\operatorname{Re} b>0$. By two applications of Theorem 2, in which $\lambda$ and $c$ are replaced by $b$ and $c$ respectively in the first, and by $c$ and $b$ respectively in the second,

$$
I^{b} H(a, b, c) f=H(a, b, b+c) f=I^{c} H(a, b, b) f .
$$

The left member is zero by (4.1); hence $H(a, b, b) f(x)={ }^{\circ} 0$ by (3.3), $H(a, b, b) f$ being locally integrable by Theorem 1 with $h=0, k=b$ and $c=b$. Now the hypergeometric function in $H(a, b, b) f$ simplifies, in fact

$$
x^{-a} \int_{0}^{x} \frac{(x-t)^{b-1}}{\Gamma(b)} t^{a} f(t) d t=H(a, b, b) f(x)={ }^{\circ} 0 .
$$

Thus $I^{b} x^{a} f(x)={ }^{\circ} 0$; and so $x^{a} f(x)={ }^{\circ} 0$ by (3.3) again, provided that $x^{a} f(x)$ is locally integrable. This is the case because, for each $l<d$,

$$
\left|x^{a} f(x)\right|=x^{\alpha}|f(x)| \leqq l^{\alpha-q} x^{q}|f(x)|
$$

if $0<x<l$ and $\alpha=\operatorname{Re} a>q$. This proves the theorem if $\operatorname{Re} b>0$.

(ii) Suppose now that $\operatorname{Re} b<\operatorname{Re} c$. Using Kummer's relation [(10), 2.9(2)], we rewrite (4.1) as

$$
\int_{0}^{x} \frac{(x-t)^{c-1}}{\Gamma(c)} F\left(c-a, c-b, c, 1-\frac{x}{t}\right) t^{a+b-c} f(t) d t={ }^{\circ} 0
$$

and also write

$$
\begin{gathered}
c-a=a^{\prime}, \quad c-b=b^{\prime}, \quad c=c^{\prime}, \\
t^{a+b-c} f(t)=f^{\prime}(t), \quad q-\alpha-\beta+\gamma=q^{\prime},
\end{gathered}
$$

where $f^{\prime}$ is given an unconventional meaning, and $\alpha, \beta, \gamma$ denote $\operatorname{Re} a, \operatorname{Re} b, \operatorname{Re} c$ respectively. The hypotheses of Theorem 4 now apply to $a^{\prime}, b^{\prime}, c^{\prime}, q^{\prime}, f^{\prime}$, and in

$\dagger$ Also called Theorem 4 A. 
addition $\operatorname{Re} b^{\prime}>0$. Consequently by case (i) of this theorem $f^{\prime}(x)={ }^{\circ} 0$, and so $f(x)={ }^{\circ} 0$ as required.

In verifying that the hypotheses apply to $a^{\prime}, b^{\prime}, c^{\prime}, q^{\prime}, f^{\prime}$ as defined by (4.4), the least straightforward step is to show that $q^{\prime} \leqq \operatorname{Re}\left(a^{\prime}+b^{\prime}\right)$. To do this we have, since $q<\beta$ and $\operatorname{Re} b^{\prime}=\operatorname{Re}(c-b)>0$,

$$
q^{\prime}<\beta-\alpha-\beta+\gamma=\gamma-\alpha=\operatorname{Re} a^{\prime}<\operatorname{Re}\left(a^{\prime}+b^{\prime}\right) .
$$

The local integrability requirement is fulfilled because $x^{q^{\prime}}\left|f^{\prime}(x)\right|=x^{q}|f(x)|$.

Remark. In case (i) the hypothesis $q \leqq \operatorname{Re}(a+b)$ is redundant; it is a consequence of the assumptions $q<\operatorname{Re} a$ and $\operatorname{Re} b>0$, and is naturally not used in the proof. In case (ii) the hypothesis $q \leqq \operatorname{Re} c$ is similarly redundant. In the case $0<\operatorname{Re} b<\operatorname{Re} c$, which is common to (i) and (ii), it follows that the hypothesis $q \leqq \min (\operatorname{Re} c, \operatorname{Re}(a+b))$ is redundant. then

Corollary 1. If $\operatorname{Re} c>0, q \leqq \min (\operatorname{Re} c, \operatorname{Re}(a+b)), q<\min (\operatorname{Re} a, \operatorname{Re} b)$,

$$
\int_{0}^{x} \frac{(x-t)^{c-1}}{\Gamma(c)} F\left(a, b, c, 1-\frac{x}{t}\right) f(t) d t={ }^{\circ} g(x) \text { in } 0<x<d
$$

has at most one solution $f$ (or rather one class of equivalent solutions) such that $x^{q} f(x)$ is locally integrable on $[0, d)$.

Corollary 2. If $\operatorname{Re} c>0$ and $0<\alpha<d$, then the solutions $f$ of

$$
\int_{\alpha}^{x} \frac{(x-t)^{c-1}}{\Gamma(c)} F\left(a, b, c, 1-\frac{x}{t}\right) f(t) d t={ }^{\circ} g(x) \text { in } \alpha<x<d
$$

which are locally integrable on $[\alpha, d)$ are the truncations, omitting $[0, \alpha)$, of the solutions $f$ of (4.5) for which $x^{q} f(x)$ is locally integrable on $[0, d)$, provided that we define $g(x)={ }^{\circ} 0$ in $0<x<\alpha$ and $q$ satisfies the hypotheses of Corollary 1.

Clearly solutions $f$ of (4.6) give, when assigned the value 0 on $(0, \alpha)$, solutions $f$ of (4.5), with $g$ defined as stated; and $x^{q} f(x)$ is integrable on $(0, l)$ for each $l<d$ since it is obtainable from integrable $f(x)$ by multiplication by a bounded function.

Conversely, solutions $f$ of (4.5) having the stated integrability satisfy

$$
\int_{0}^{x} \frac{(x-t)^{c-1}}{\Gamma(c)} F\left(a, b, c, 1-\frac{x}{t}\right) f(t) d t={ }^{\circ} 0 \text { in } 0<x<\alpha,
$$

and $x^{q} f(x)$ is locally integrable on $[0, \alpha)$. So by Theorem 4 they vanish almost everywhere in $(0, \alpha)$, and therefore satisfy $(4.6)$ when truncated. Also they are integrable on $(\alpha, l)$, for each $l$ such that $\alpha<l<d$, because they are obtainable from the integrable $x^{4} f(x)$ by multiplication by a bounded function.

\section{Digression on $I^{b} x^{a} f(x)=x^{a} I^{b} g(x)$}

This section can be read independently of the rest of the paper, except for 
Theorem 1. Its subject is the integral equation abbreviated in the title, namely

$$
\int_{0}^{x} \frac{(x-t)^{b-1}}{\Gamma(b)} t^{a} f(t) d t={ }^{\circ} x^{a} \int_{0}^{x} \frac{(x-t)^{b-1}}{\Gamma(b)} g(t) d t
$$

with either $f$ or $g$ regarded as unknown function. Formal solution by fractional differentiation is easily done; and uniqueness of solutions is immediate from (3.3). The interest is not in these things, but in conditions for existence of solutions.

Only Lemma 2 of this section is needed in $\S 6$, where we consider necessary conditions for existence of solutions of (1.4). Theorem 5 is used in establishing sufficient conditions in $\S 7$ and $\S 8$, and Theorem 6 in making some of the refinements given in $\S 9$.

We begin with Lemma 2, which may be regarded as a limiting case $c=0$ of Lemma 1 on Bateman's integral; however an independent proof is simpler.

Lemma 2. If $\operatorname{Re} c>0$ and $z$ is in the complex plane cut along $z \geqq 1$, then

$$
\frac{1}{\Gamma(c)}\{F(a, b, c, z)-1\}=a b z \int_{0}^{1} \frac{(1-u)^{c-1}}{\Gamma(c)} F(a+1, b+1,2, z u) d u .
$$

In particular, if $\operatorname{Re} c>0$ and $0<t<x$, then

$$
\begin{aligned}
\frac{(x-t)^{c-1}}{\Gamma(c)}\left\{F\left(a, b, c, 1-\frac{x}{t}\right)-1\right\} \\
=-\frac{a b}{t} \int_{t}^{x} \frac{(x-s)^{c-1}}{\Gamma(c)} F\left(a+1, b+1,2,1-\frac{s}{t}\right) d s .
\end{aligned}
$$

First suppose that $|z|<1$. Then the hypergeometric series with variable $z u$ is boundedly convergent on $0 \leqq u \leqq 1$, and so the right side of (5.2) is equal to

$$
\begin{aligned}
a b z & \sum_{n=0}^{\infty} \frac{(a+1)_{n}(b+1)_{n}}{(2)_{n}} z^{n} \int_{0}^{1} \frac{(1-u)^{c-1}}{\Gamma(c)} \frac{u^{n}}{n !} d u \\
& =\sum_{n=0}^{\infty} \frac{(a)_{n+1}(b)_{n+1}}{(n+1) !} \frac{z^{n+1}}{\Gamma(c+n+1)}=\frac{1}{\Gamma(c)} \sum_{n=0}^{\infty} \frac{(a)_{n+1}(b)_{n+1}}{(c)_{n+1}} \frac{z^{n+1}}{(n+1) !}
\end{aligned}
$$

as required. Further, both sides of (5.2) are regular functions of $z$ in the cut plane, by the usual arguments; so they are equal throughout it.

The particular case (5.3) is obtained by the substitutions

$$
z=\frac{t-x}{t} \text { and } u=\frac{s-t}{x-t}
$$

Remark. A similar integral can be obtained for the difference between the hypergeometric function and the $m$ th partial sum of the hypergeometric series.

Definition. $Q_{q}$ is the class of all functions $f$ such that $x^{q} f(x)$ is locally integrable on $[0, d)$. 
Theorem 5. If $q<0<\operatorname{Re} b$ and $q<\operatorname{Re} a$, then the equation (5.1), that is

$$
I^{b} x^{a} f(x)={ }^{\circ} x^{a} I^{b} g(x) \text { in } 0<x<d,
$$

has, for each $f$ in $Q_{q}$ a solution $g$ in $Q_{q}$, and for each $g$ in $Q_{q}$ a solution $f$ in $Q_{q}$.

(i) Suppose $f \in Q_{q}$ is given. By Lemma 2 (5.3) with $c=b$,

$$
\begin{array}{rl}
\int_{0}^{x} \frac{(x-t)^{b-1}}{\Gamma(b)} \cdot\left(1-\frac{t^{a}}{x^{a}}\right) f(t) d t & \\
\therefore a & a b \int_{0}^{x} \frac{f(t)}{t} d t \int_{t}^{x} \frac{(x-s)^{b-1}}{\Gamma(b)} F\left(a+1, b+1,2,1-\frac{s}{t}\right) d s \\
& =a b \int_{0}^{x} \frac{(x-s)^{b-1}}{\Gamma(b)} d s \int_{0}^{s} F\left(a+1, b+1,2,1-\frac{s}{t}\right) \frac{f(t)}{t} d t
\end{array}
$$

the inversion of order of integration being justified if the last double integral is absolutely convergent, and the existence of the single integral on the left being also ensured thereby. This absolute convergence is ensured, for almost all $x$ in $(0, d)$, by the integrability on $0<s<x$ of

$$
\int_{0}^{s}\left|F\left(a+1, b+1,2,1-\frac{s}{t}\right) \frac{f(t)}{t}\right| d t
$$

and this integrability follows from Theorem 1 with $h=0, k=1, q$ replaced by $q+1$ and $f(x)$ by $x^{-1} f(x)$; for $x^{q+1} \cdot x^{-1} f(x)$ is locally integrable on $[0, d)$, $q+1<\min (\operatorname{Re}(a+1), \operatorname{Re}(b+1))$, and $q+1 \leqq 1$.

Since also $f(x)=x^{-q} \cdot x^{q} f(x)$ and $x^{-q}$ is locally bounded on $[0, d), f$ is locally integrable; thus $I^{b} f(x)$ exists for almost all $x$ and we can write (5.6) as the difference of two integrals. This gives

$$
\begin{aligned}
& \int_{0}^{x} \frac{(x-t)^{b-1}}{\Gamma(b)} f(t) d t-x^{-a} \int_{0}^{x} \frac{(x-t)^{b-1}}{\Gamma(b)} t^{a} f(t) d t \\
& \quad{ }^{\circ} a b I^{b}\left\{\int_{0}^{x} F\left(a+1, b+1,2,1-\frac{x}{t}\right) \frac{f(t)}{t} d t\right\} .
\end{aligned}
$$

and so

$$
x^{-a} I^{b} x^{a} f(x)={ }^{\circ} I^{b}\left\{f(x)-a b \int_{0}^{x} F\left(a+1, b+1,2,1-\frac{x}{t}\right) \frac{f(t)}{t} d t\right\}
$$

This shows that (5.5) is satisfied by

$$
g(x)=f(x)-a b \int_{0}^{x} F\left(a+1, b+1,2,1-\frac{x}{t}\right) \frac{f(t)}{t} d t .
$$

We have shown that the two terms on the right of (5.8) are locally integrable, and it remains to show that $g \in Q_{q}$. For this,

$$
x^{q} \int_{0}^{x} F\left(a+1, b+1,2,1-\frac{x}{t}\right) \frac{f(t)}{t} d t
$$


is locally integrable on $[0, d)$ by Theorem 1 with $h=q, k=1, q$ replaced by $q+1$ and $f(x)$ by $x^{-1} f(x)$; the treatment required is almost the same as that used for (5.7). Also $x^{q} f(x)$ is locally integrable on $[0, d)$; hence, using (5.8), $x^{q} g(x)$ is locally integrable on $[0, d)$, as required.

(ii) For the converse, suppose $g \in Q_{q}$ is given. Put $q-\operatorname{Re} a=q^{\prime},-a=a^{\prime}$, $b=b^{\prime}$ and $x^{a} g(x)=f^{\prime}(x)$ (not the derivative of $f$ ). Then

$$
q^{\prime}<0<\operatorname{Re} b^{\prime}, q^{\prime}<\operatorname{Re} a^{\prime}, \text { and } f^{\prime} \in Q_{q^{\prime}} \text {. }
$$

So by (i) there is $g^{\prime} \in Q_{q^{\prime}}$, satisfying $I^{b^{\prime}} x^{a^{\prime}} f^{\prime}(x)={ }^{\circ} x^{a^{\prime}} I^{b^{\prime}} g^{\prime}(x)$; that is, satisfying

$$
x^{-a} I^{b} g^{\prime}(x)={ }^{\circ} I^{b} g(x) \text {. }
$$

Defining $f(x)=x^{-a} g^{\prime}(x)$ we see that $x^{q}|f(x)|=x^{q^{\prime}}\left|g^{\prime}(x)\right|$, so that $f \in Q_{q}$; and

$$
I^{b} x^{a} f(x)={ }^{\circ} x^{a} I^{b} g(x) \text {. }
$$

Remark 1. The solution $f$ in (ii) can be made explicit with the help of (5.8).

$$
\begin{aligned}
f(x) & =x^{-a} g^{\prime}(x) \\
& =x^{-a}\left\{f^{\prime}(x)-a^{\prime} b^{\prime} \int_{0}^{x} F\left(a^{\prime}+1, b^{\prime}+1,2,1-\frac{x}{t}\right) \frac{f^{\prime}(t)}{t} d t\right\} \\
& =g(x)+a b x^{-a} \int_{0}^{x} F\left(1-a, 1+b, 2,1-\frac{x}{t}\right) t^{a-1} g(t) d t \\
& =g(x)+\frac{a b}{x} \int_{0}^{x} F\left(1-a, 1-b, 2,1-\frac{t}{x}\right) g(t) d t .
\end{aligned}
$$

The last step is made using $[(\mathbf{1 0}), 2.9(3)]$.

Remark 2. Both parts of the theorem are false if $q>0$, as the following examples show.

(i) If $b=1$ and $f(x)=x^{-\frac{1}{2} q-1}$, clearly $f \in Q_{q}$. And since $\operatorname{Re} a>q>\frac{1}{2} q$,

$$
x^{-a} I^{b} x^{a} f(x)=\frac{x^{-\frac{1}{2} q}}{a-\frac{1}{2} q}
$$

this is not expressible as $I^{b} g(x)$ because it is unbounded near 0 .

(ii) If $b=1$ and $g(x)=x^{-\frac{1}{2} q-1}$, clearly $g \in Q_{q}$ but $I^{b} g$ does not exist.

Remark 3. Part (i) of the theorem remains true if $q=0$, the proof being unchanged; but part (ii) becomes false. The proof fails because $q^{\prime}=\operatorname{Re} a^{\prime}$ occurs in (5.9); and the following example shows that part (ii) itself is false.

Let $b=1$ and $g(x)=1 / x\{\log (3 / x)\}^{2}$ if $0<x<1, g(x)=0$ if $x \geqq 1$. Since $\operatorname{Re} a>0$ we have, if $0<x<1$,

$$
\begin{gathered}
x^{a} I^{b} g(x)=\frac{x^{a}}{\log (3 / x)}=I^{b}\left(\frac{a x^{a-1}}{\log (3 / x)}+\frac{x^{a-1}}{\{\log (3 / x)\}^{2}}\right), \\
x^{-a} I^{-b} x^{a} I^{b} g(x)=\frac{a}{x \log (3 / x)}+\frac{1}{x\{\log (3 / x)\}^{2}} .
\end{gathered}
$$


This is the only possibility for a solution $f$, and it is not in $Q_{0}$ because it is not locally integrable on $[0, d)$.

Theorem 6 below is a replacement for Theorem 5 when $q=0$. To shorten the proof it is convenient to prove first the following lemma.

Lemma 3. If $\operatorname{Re} a>0, \operatorname{Re} b>0$ and $h$ is integrable on $(0, l)$, then

$$
\frac{1}{x^{2}} \int_{0}^{x}\left|F\left(2-a, 2-b, 3,1-\frac{t}{x}\right) \operatorname{th}(t)\right| d t
$$

is also integrable on $(0, l)$.

First suppose that $\operatorname{Re}(a+b)>1$. Then there is $M$, independent of $u$, such that

$$
|F(2-a, 2-b, 3, u)| \leqq M \text { for } 0 \leqq u \leqq 1,
$$

since this hypergeometric function is continuous at $u=1$, by Abel's continuity theorem and [(10), 2.1(14)]. So

$$
\begin{aligned}
\int_{0}^{t} \frac{d x}{x^{2}} \int_{0}^{x} \mid F & \left(2-a, 2-b, 3,1-\frac{t}{x}\right) \operatorname{th}(t) \mid d t \\
& \leqq M \int_{0}^{t} \frac{d x}{x^{2}} \int_{0}^{x} t|h(t)| d t=M \int_{0}^{t} t|h(t)| d t \int_{t}^{t} \frac{d x}{x^{2}} \leqq M \int_{0}^{t}|h(t)| d t,
\end{aligned}
$$

and the finiteness of the last integral gives the result.

Now suppose that $\operatorname{Re}(a+b) \leqq 1$. Writing $\alpha$ and $\beta$ for $\operatorname{Re} a$ and $\operatorname{Re} b$, we have $0<\alpha<1$ and $0<\beta<1$ as well as $\alpha+\beta \leqq 1$. Since $3>2-\beta>0$, Euler's integral [(10), 2.1(10)] gives, if $u<1$,

$$
\begin{aligned}
|F(2-a, 2-b, 3, u)| & =\left|\frac{\Gamma(3)}{\Gamma(2-b) \Gamma(1+b)} \int_{0}^{1} s^{1-b}(1-s)^{b}(1-s u)^{a-2} d s\right| \\
& \leqq M^{\prime} \int_{0}^{1} s^{1-\beta}(1-s)^{\beta}(1-s u)^{\alpha-2} d s
\end{aligned}
$$

where $M^{\prime}$ depends only on $b$. Now $(1-s u)^{\alpha-2} \leqq(1-s)^{-1}(1-u)^{\alpha-1}$ when $0<u<1$ and $0<s<1$, because $\alpha<1$; so we have

when $0<u<1$. Thus

$$
\begin{aligned}
|F(2-a, 2-b, 3, u)| & \leqq M^{\prime} \int_{0}^{1} s^{1-\beta}(1-s)^{\beta-1}(1-u)^{\alpha-1} d s \\
& \leqq M^{\prime}(1-u)^{\alpha-1} \int_{0}^{1}(1-s)^{\beta-1} d s=\frac{M^{\prime}}{\beta}(1-u)^{\alpha-1}
\end{aligned}
$$

$$
\begin{aligned}
\int_{0}^{t} \frac{d x}{x^{2}} \int_{0}^{x}\left|F\left(2-a, 2-b, 3,1-\frac{t}{x}\right) \operatorname{th}(t)\right| d t \\
\quad \leqq \frac{M^{\prime}}{\beta} \int_{0}^{l} \frac{d x}{x^{2}} \int_{0}^{x} \frac{t^{\alpha}}{x^{\alpha-1}}|h(t)| d t \\
\quad=\frac{M^{\prime}}{\beta} \int_{0}^{l} t^{\alpha}|h(t)| d t \int_{t}^{t} \frac{d x}{x^{\alpha+1}} \leqq \frac{M^{\prime}}{\alpha \beta} \int_{0}^{l}|h(t)| d t ;
\end{aligned}
$$

and the finiteness of the last integral again gives the result. 
Definitions. $Q_{0}$ is the class of functions which are locally integrable on $[0, d)$; and, for $g \in Q_{0}, \bar{g}$ is the function whose values are given by

$$
\bar{g}(x)=\frac{1}{x} \int_{0}^{x} g(t) d t .
$$

The example in remark 3 above shows that $g \in Q_{0}$ does not ensure that $\bar{g} \in Q_{0}$. We shall see in Lemma 6, however, that $g \in Q_{q}$ implies that $\bar{g} \in Q_{q}$ if $q<0$.

Theorem 6. If $\operatorname{Re} a>0$ and $\operatorname{Re} b>0$, then the equation (5.1), that is

$$
I^{b} x^{a} f(x)={ }^{\circ} x^{a} I^{b} g(x) \text { in } 0<x<d,
$$

has, for each $f$ in $Q_{0}$, a solution $g$ such that $g$ and $\bar{g}$ are in $Q_{0}$; and conversely for each $g$ such that $g$ and $\bar{g}$ are in $Q_{0}$, a solution $f$ in $Q_{0}$.

(i) Suppose $f \in Q_{0}$ is given. Proof (i) of Theorem 5, with $q=0$, still establishes a solution $g \in Q_{0}$ expressed by (5.8). We show that $\bar{g} \in Q_{0}$.

Since $f \in Q_{0}$ we have, for $0<x<d$,

$$
\begin{aligned}
\bar{g}(x) & =\frac{1}{x} \int_{0}^{x} f(s) d s-\frac{a b}{x} \int_{0}^{x} d s \int_{0}^{s} F\left(a+1, b+1,2,1-\frac{s}{t}\right) \frac{f(t)}{t} d t \\
& =\circ \frac{1}{x} \int_{0}^{x} f(s) d s-\frac{a b}{x} \int_{0}^{x} \frac{f(t)}{t} d t \int_{t}^{x} F\left(a+1, b+1,2,1-\frac{s}{t}\right) d s
\end{aligned}
$$

by the same argument as was used about (5.7). By Lemma 2 with $c=1$,

$$
\begin{aligned}
\bar{g}(x) & =\circ \frac{1}{x} \int_{0}^{x} f(s) d s+\frac{1}{x} \int_{0}^{x} f(t)\left\{F\left(a, b, 1,1-\frac{x}{t}\right)-1\right\} d t \\
& =\frac{1}{x} \int_{0}^{x} F\left(a, b, 1,1-\frac{x}{t}\right) f(t) d t .
\end{aligned}
$$

Thus $\bar{g}$ is locally integrable on $[0, d)$ as required, by Theorem 1 with $h=-1$, $k=1, q=0, c=1$.

(ii) Suppose $g$ is given, such that $g \in Q_{0}$ and $\bar{g} \in Q_{0}$. We prove that

$$
f(x)=g(x)+\frac{a b}{x} \int_{0}^{x} F\left(1-a, 1-b, 2,1-\frac{t}{x}\right) g(t) d t
$$

suggested by (5.10), defines a function $f$ which is locally integrable and satisfies (5.14).

Since $\operatorname{Re}\{2-(1-a)-(1-b)\}=\operatorname{Re}(a+b)>0, \quad F(1-a, 1-b, 2, u)$ is continuous in $0 \leqq u \leqq 1$, using Abel's continuity theorem and [(10), 2.1(14)]. Since also $g \in Q_{0}$, the integral in (5.15) exists for $0<x<d$. So (5.15) does define a function $f$.

Integrating by parts in (5.15), as we may since the hypergeometric factor has 
continuous derivative with respect to $t$ in $0<t \leqq x$; and using (5.13),

$$
\begin{aligned}
f(x) & =g(x)+\frac{a b}{x}\left\{x \bar{g}(x)-\int_{0}^{x} t \bar{g}(t) \frac{\partial}{\partial t} F\left(1-a, 1-b, 2,1-\frac{t}{x}\right) d t\right\} \\
& =g(x)+a b \bar{g}(x)+\frac{a b(1-a)(1-b)}{2 x^{2}} \int_{0}^{x} t \bar{g}(t) F\left(2-a, 2-b, 3,1-\frac{t}{x}\right) d t .
\end{aligned}
$$

All three terms of this expression are locally integrable; the first two by data, and the integral term by Lemma 3 with $h$ replaced by $\bar{g}$. So $f \in Q_{0}$.

Finally, it remains to prove that (5.15) satisfies (5.14). Since $\operatorname{Re} a>0$, $x^{a} f(x)$ and $x^{a} g(x)$ are locally integrable; so $I^{b} x^{a} f(x)$ and $I^{b} x^{a} g(x)$ exist almost everywhere and, by (5.15),

$$
\begin{aligned}
I^{b} x^{a} f(x)-I^{b} x^{a} g(x) & =\int_{0}^{x} \frac{(x-s)^{b-1}}{\Gamma(b)} a b s^{a-1} d s \int_{0}^{s} F\left(1-a, 1-b, 2,1-\frac{t}{s}\right) g(t) d t \\
& =a b \int_{0}^{x} g(t) d t \int_{t}^{x} \frac{(x-s)^{b-1}}{\Gamma(b)} s^{a-1} F\left(1-a, 1-b, 2,1-\frac{t}{s}\right) d s
\end{aligned}
$$

this double integral being absolutely convergent because the hypergeometric function is bounded for $0<t \leqq s$, as above, and because

$$
\begin{aligned}
\int_{0}^{x}|g(t)| d t \int_{t}^{x}\left|(x-s)^{b-1} s^{a-1}\right| d s & \leqq \int_{0}^{x}|g(t)| d t \int_{0}^{x}(x-s)^{\beta-1} s^{\alpha-1} d s \\
& =\int_{0}^{x}|g(t)| d t \frac{\Gamma(\alpha) \Gamma(\beta)}{\Gamma(\alpha+\beta)} x^{\alpha+\beta-1},
\end{aligned}
$$

which is finite for $0<x<d ; \alpha$ and $\beta$ denoting $\operatorname{Re} a$ and $\operatorname{Re} b$ as usual. Thus, using [(10), 2.9(3)], and then Lemma 2 with $a$ and $c$ replaced by $-a$ and $b$,

$$
\begin{aligned}
I^{b} x^{a} f(x) & -I^{b} x^{a} g(x) \\
& ={ }^{o} a b \int_{0}^{x} t^{a-1} g(t) d t \int_{t}^{x} \frac{(x-s)^{b-1}}{\Gamma(b)} F\left(1-a, 1+b, 2,1-\frac{s}{t}\right) d s \\
& =\int_{0}^{x} t^{a} g(t) \frac{(x-t)^{b-1}}{\Gamma(b)}\left\{F\left(-a, b, b, 1-\frac{x}{t}\right)-1\right\} d t \\
& =\int_{0}^{x} \frac{(x-t)^{b-1}}{\Gamma(b)} g(t) t^{a}\left\{\left(\frac{x}{t}\right)^{a}-1\right\} d t \\
& ={ }^{\circ} x^{a} I^{b} g(x)-I^{b} x^{a} g(x) .
\end{aligned}
$$

This proves that (5.15) satisfies (5.14), and the whole theorem is proved.

6. Necessary conditions for existence of solutions of (1.4)

Theorem 7. If $\operatorname{Re} c>0, q \leqq 0, q<\min (\operatorname{Re} a, \operatorname{Re} b)$, $f$ is in $Q_{q}$ and

$$
\int_{0}^{x} \frac{(x-t)^{c-1}}{\Gamma(c)} F\left(a, b, c, 1-\frac{x}{t}\right) f(t) d t={ }^{\circ} g(x)
$$

then $I^{-c} g$ exists and is in $Q_{q}$.

E.M.S. $-\mathbf{N}$ 
Our proof is very like that of Theorem 5 (i). By Lemma 2 (5.3),

$$
\begin{aligned}
& \int_{0}^{x} \frac{(x-t)^{c-1}}{\Gamma(c)}\left\{F\left(a, b, c, 1-\frac{x}{t}\right)-1\right\} f(t) d t \\
& =-a b \int_{0}^{x} \frac{f(t)}{t} d t \int_{t}^{x} \frac{(x-s)^{c-1}}{\Gamma(c)} F\left(a+1, b+1,2,1-\frac{s}{t}\right) d s \\
& =-a b \int_{0}^{x} \frac{(x-s)^{c-1}}{\Gamma(c)} d s \int_{0}^{s} F\left(a+1, b+1,2,1-\frac{s}{t}\right) \frac{f(t)}{t} d t,
\end{aligned}
$$

the inversion of order of integration being justified if the last double integral is absolutely convergent. This is ensured, for almost all $x$ in $(0, d)$, by the integrability of (5.7) established in proof (i) of Theorem 5; this is valid for $q=0$ as well as for $q<0$.

Again $f(x)=x^{-q} \cdot x^{q} f(x)$ is locally integrable; thus $I^{c} f(x)$ exists for almost all $x$, by (3.1). So (6.2) can be written as the difference of two integrals existing almost everywhere, and we obtain

$$
\begin{aligned}
\int_{0}^{x} \frac{(x-t)^{c-1}}{\Gamma(c)} & F\left(a, b, c, 1-\frac{x}{t}\right) f(t) d t \\
& =\int_{0}^{x} \frac{(x-s)^{c-1}}{\Gamma(c)}\left\{f(s)-a b \int_{0}^{s} F\left(a+1, b+1,2,1-\frac{s}{t}\right) \frac{f(t)}{t} d t\right\} d s .
\end{aligned}
$$

Taking account of (6.1), this equation is $g(x)={ }^{\circ} I^{c} \phi(x)$, where

$$
\phi(x)=f(x)-a b \int_{0}^{x} F\left(a+1, b+1,2,1-\frac{x}{t}\right) \frac{f(t)}{t} d t,
$$

and the two terms on the right have already been proved locally integrable. So $\phi$ is locally integrable, and thus $I^{-c} g$ exists.

Further $x^{q} f(x)$ is locally integrable by data; and

$$
x^{q} \int_{0}^{x} F\left(a+1, b+1,2,1-\frac{x}{t}\right) \frac{f(t)}{t} d t
$$

is locally integrable, by Theorem 1 with $h=q, k=1, c=2$, and $q, a, b, f(x)$ replaced by $q+1, a+1, b+1, x^{-1} f(x)$ respectively. So, noticing $(6.3), x^{q} \phi(x)$ is locally integrable, that is, $I^{-c} g \in Q_{q}$ as required.

Remark 1. The hypothesis $q \leqq 0$ is necessary; it cannot be replaced by $q \leqq \operatorname{Re} c$, as might be suggested by a glance at Theorem 4 . For if

$$
0<q<a<1=b=c \text { and } f(x)=x^{-\frac{1}{2} q-1},
$$

all hypotheses of Theorem 7 except $q \leqq 0$ are satified, but

$$
g(x)=0 \int_{0}^{x}\left(\frac{x}{t}\right)^{-a} t^{-\frac{1}{2} q-1} d t=x^{-a} \int_{0}^{x} t^{a-\frac{1}{2} q-1} d t=\frac{x^{-\frac{1}{2} q}}{a-\frac{1}{2} q} .
$$

This is unbounded in every neighbourhood of 0 , and hence is not expressible as $I^{c} \phi(x)$ for any locally integrable $\phi(x)$. 
Remark 2. Under the conditions of Theorem 7, equation (6.1) is equivalent to the integral equation of the second kind

$$
f(x)-a b \int_{0}^{x} F\left(a+1, b+1,2,1-\frac{x}{t}\right) \frac{f(t)}{t} d t={ }^{\circ} I^{-c} g(x) .
$$

This clearly invites generalisation.

\section{Sufficient conditions for existence of solutions of (1.4)}

In Theorem 4 on uniqueness, the proof was given in two parts, (i) for the case $\operatorname{Re} b>0$ and (ii) for the case $\operatorname{Re} b<\operatorname{Re} c$. In considering existence it seems preferable to separate these two cases into different theorems; for not only are different conditions appropriate in the two cases, but also different explicit solution formulae. Theorems 8 and 9 are these two theorems. Since the distinction between them is unsymmetrical in $a$ and $b$, it is not surprising that their hypotheses are unsymmetrical also. Theorem 10 is a composite version of them which is symmetrical in $a$ and $b$. It gives sufficient conditions very little more restrictive than the necessary conditions given in Theorem 7 .

These theorems involve a few simple results on fractional integrals of purely imaginary order. Some of these are proved as they arise in the course of other proofs, for lack of a suitable reference. Others are collected in Lemma 5.

Lemma 4. If $\operatorname{Re} b>0, \operatorname{Re} c>0, q \leqq \operatorname{Re} c, q<\min (\operatorname{Re} a, \operatorname{Re} b)$, and $f$ is in $Q_{q}$, then (see definitions at (5.4), (3.5) and (3.1))

$$
H(a, b, c) f(x)={ }^{o} I^{c-b} x^{-a} I^{b} x^{a} f(x) .
$$

If $\operatorname{Re} b=\operatorname{Re} c$ the meaning of $I^{c-b}$ is $I^{-1} I^{1+c-b}$, where $I^{-1}$ denotes ordinary differentiation.

By two applications of Theorem 2, in which $\lambda$ and $c$ are replaced by $b$ and $c$ in the first application, and by $c$ and $b$ in the second,

But

$$
I^{b} H(a, b, c) f=H(a, b, b+c) f=I^{c} H(a, b, b) f .
$$

$$
H(a, b, b) f(x)=\int_{0}^{x} \frac{(x-t)^{b-1}}{\Gamma(b)}\left(\frac{x}{t}\right)^{-a} f(t) d t=x^{-a} I^{b} x^{a} f(x),
$$

as in (4.2); so

$$
I^{b} H(a, b, c) f(x)={ }^{\circ} I^{c} x^{-a} I^{b} x^{a} f(x) .
$$

If $\operatorname{Re} c>\operatorname{Re} b$ we have $I^{c}=I^{b} I^{c-b}$ as in (3.2), and so

$$
I^{b}\left\{H(a, b, c) f(x)-I^{c-b} x^{-a} I^{b} x^{a} f(x)\right\}={ }^{\circ} 0 .
$$

From this (7.1) follows by (3.3). For $H(a, b, c) f$ and $H(a, b, b) f$ are locally integrable by the above applications of Theorem 2 , and consequently

$$
I^{c-b} H(a, b, b) f
$$

is locally integrable. Noticing (7.3), this shows that the two terms bracketed in (7.5) are locally integrable, which justifies the application of (3.3). 
If $\operatorname{Re} c<\operatorname{Re} b$ we have $I^{b}=I^{c} I^{b-c}$ as in (3.2), and so (7.4) gives

$$
I^{c}\left\{I^{b-c} H(a, b, c) f(x)-x^{-a} I^{b} x^{a} f(x)\right\}={ }^{\circ} 0 .
$$

From this (7.1) again follows by (3.3), the bracketed terms having the requisite local integrability as in the preceding paragraph.

If $\operatorname{Re} c=\operatorname{Re} b$, choose an integer $m>\operatorname{Re} b$. Then, by (3.2), $I^{m-b} I^{b}=I^{m}$ and $I^{m-b} I^{c}=I^{m-b+c}$, so (7.4) gives

$$
I^{m} H(a, b, c) f(x)={ }^{\circ} I^{m-b+c} x^{-a} I^{b} x^{a} f(x) .
$$

This equation actually holds throughout $(0, d)$ because both sides are continuous, the integer $m$ being necessarily positive. Similarly at each of $m-1$ differentiations, which lead to

$$
I^{1} H(a, b, c) f(x)=I^{1+c-b} x^{-a} I^{b} x^{a} f(x) .
$$

The left side is locally absolutely continuous, hence so are both sides. Thus a final differentiation gives (7.1) almost everywhere, with $I^{-1} I^{1+c-b}$ in place of $I^{c-b} ; I^{-1}$ denoting $d / d x$.

Theorem 8. If $\operatorname{Re} c>0, \operatorname{Re} b>0, q<\min (0, \operatorname{Re} a)$ and $I^{-c} g$ exists in $Q_{q}$ (defined at (5.4)), then the equation

$$
\int_{0}^{x} \frac{(x-t)^{c-1}}{\Gamma(c)} F\left(a, b, c, 1-\frac{x}{t}\right) f(t) d t={ }^{\circ} g(x)
$$

has a solution $f$ in $Q_{q}$, expressible by

$$
f(x)=x^{-a} I^{-b} x^{a} I^{b-c} g(x) .
$$

If $\operatorname{Re} b=\operatorname{Re} c$ the meaning of $I^{b-c}$ is $I^{-1} I^{1+b-c}$; equivalent expressions are $I^{b} I^{-c}$ and $I^{-c} I^{b}$, and others obtainable from Lemma 5 (below).

Let $I^{-c} g=h$; by definition $h$ is locally integrable, so $I^{b} h$ is also. We first prove that

$$
I^{b-c} g=I^{b} h .
$$

If $\operatorname{Re} b>\operatorname{Re} c$ then, by (3.2), $I^{b} h=I^{b-c} I^{c} h=I^{b-c} g$, giving (7.8).

If $\operatorname{Re} b<\operatorname{Re} c$ then, by (3.2),g $=I^{c} h=I^{c-b} I^{b} h$; this gives (7.8) by inverting the operator $I^{c-b}$.

If $\operatorname{Re} b=\operatorname{Re} c$ then, using (3.2) twice,

$$
I^{1+b-c} g=I^{1+b-c} I^{c} h=I^{1+b} h=I^{1} I^{b} h ;
$$

since $I^{b} h$ is locally integrable, differentiation almost everywhere gives

$$
I^{-1} I^{1+b-c} g=I^{b} h
$$

which is (7.8) with the stated meaning of $I^{b-c} g$.

Since $h=I^{-c} g \in Q_{q}$, Theorem 5 (ii) shows that the equation

$$
I^{b} x^{a} f(x)={ }^{o} x^{a} I^{b} h(x)
$$

has a solution $f \in Q_{q}$. This solution is (7.7); for $x^{a} f(x)=x^{a-q} x^{q} f(x)$ is locally 
integrable and so, using (7.9) and (7.8),

$$
f(x)=x^{-a} I^{-b} x^{a} I^{b} h(x)=x^{-a} I^{-b} x^{a} I^{b-c} g(x) .
$$

Again, (7.9) and (7.8) give

whence

$$
x^{-a} I^{b} x^{a} f(x)={ }^{o} I^{b} h(x)=I^{b-c} g(x) .
$$

$$
I^{c-b} x^{-a} I^{b} x^{a} f(x)={ }^{\circ} g(x),
$$

at least if $\operatorname{Re} b \neq \operatorname{Re} c$. And if $\operatorname{Re} b=\operatorname{Re} c$ we have, using (3.2) twice,

$$
g=I^{-1} I^{1} g=I^{-1} I^{1} I^{c} h=I^{-1} I^{1+c} h=I^{-1} I^{1+c-b} I^{b} h=I^{c-b} I^{b} h ;
$$

this inverts (7.8), and from it (7.10) follows using (7.9).

The hypotheses of Lemma 4 are fulfilled, with $f \in Q_{q}$ given by (7.7). So, putting (7.1) of Lemma 4 and (7.10) together, $f$ satisfies (7.6) as required.

Theorem 9. If $\operatorname{Re} c>0, \operatorname{Re} b<\operatorname{Re} c, q<\min (\operatorname{Re}(a+b-c), \operatorname{Re} b)$ and $x^{-a} I^{-c} x^{a} g(x)$ exists in $Q_{q}$, then

$$
\int_{0}^{x} \frac{(x-t)^{c-1}}{\Gamma(c)} F\left(a, b, c, 1-\frac{x}{t}\right) f(t) d t={ }^{\circ} g(x)
$$

has a solution $f$ in $Q_{q}$, expressible by

$$
f(x)=x^{-b} I^{b-c} x^{c-a} I^{-b} x^{a+b-c} g(x) .
$$

If $\operatorname{Re} b=0$ the meaning of $I^{-b}$ is $I^{-1} I^{1-b}$; equivalent expressions are $I^{c-b} I^{-c}$ and $I^{-c} I^{c-b}$, and others obtainable from Lemma 5.

Using $[(10), 2.9(2)]$, equation (7.11) is equivalent to

$$
\int_{0}^{x} \frac{(x-t)^{c-1}}{\Gamma(c)} F\left(a^{\prime}, b^{\prime}, c, 1-\frac{x}{t}\right) f^{\prime}(t) d t={ }^{\circ} g^{\prime}(x),
$$

where

$$
\left.\begin{array}{r}
f^{\prime}(x)=x^{a+b-c} f(x), g^{\prime}(x)=x^{a+b-c} g(x), \\
a^{\prime}=c-a, \quad b^{\prime}=c-b, \quad q^{\prime}=q+\gamma-\alpha-\beta,
\end{array}\right\}
$$

and we have written $\alpha, \beta, \gamma$ for $\operatorname{Re} a, \operatorname{Re} b, \operatorname{Re} c$ respectively. We now verify that Theorem 8 with accented symbols applies to (7.13).

The only items in this verification which are not immediate are that $I^{-c} g^{\prime}$ exists and is in $Q_{q}$. Putting $h(x)=I^{-c} x^{a} g(x)$, we have by data that $x^{-a} h(x)$ exists in $Q_{q}$, and so $h \in Q_{q-a}$. Now the equation

$$
I^{c} x^{b-c} \phi(x)={ }^{\circ} x^{b-c} I^{c} h(x)
$$

is satisfied by some $\phi \in Q_{q-a}$; because $q-\alpha<\beta-\gamma<0<\gamma$, which justifies the application of Theorem 5 (ii) to (7.15). Thus

$$
I^{c} x^{b-c} \phi(x)={ }^{\circ} x^{b-c} x^{a} g(x)=g^{\prime}(x) .
$$

Further $x^{b-c} \phi(x) \in Q_{q-\alpha-\beta+\gamma}=Q_{q^{\prime}} \subset Q_{0}$ since $q^{\prime}=q-\alpha-\beta+\gamma<0$. Thus $I^{-c} g^{\prime}(x)$ both exists and is in $Q_{q}$.

So Theorem 8 with accented symbols is applicable to (7.13), which therefore 
has a solution $f^{\prime}$ in $Q_{q^{\prime}}$ expressible by

$$
f^{\prime}(x)=x^{-a^{\prime}} I^{-b^{\prime}} x^{a^{\prime}} I^{b^{\prime}-c} g^{\prime}(x),
$$

with $I^{b^{\prime}-c}$ meaning $I^{-1} I^{1+b^{\prime}-c}$ if $\operatorname{Re}\left(b^{\prime}-c\right)=0$. Translating this by means of (7.14) we obtain the stated conclusions.

Theorem 10. If $\operatorname{Re} c>0, q<\min (0, \operatorname{Re}(a+b-c)), q<\min (\operatorname{Re} a, \operatorname{Re} b)$, and $I^{-c} g$ exists in $Q_{q}$, then the equation

$$
\int_{0}^{x} \frac{(x-t)^{c-1}}{\Gamma(c)} F\left(a, b, c, 1-\frac{x}{t}\right) f(t) d t={ }^{\circ} g(x)
$$

has a unique solution $f$ in $Q_{q}$, given by (7.7) if $\operatorname{Re} b>0$ and by (7.12) if $\operatorname{Re} b<\operatorname{Re} c$.

The uniqueness follows from Theorem 4. If $\operatorname{Re} b>0$ the rest follows from Theorem 8. It remains to show that the hypotheses of Theorem 9 are fulfilled if $\operatorname{Re} b<\operatorname{Re} c$, and then the rest will follow from Theorem 9 .

Let $\psi=I^{-c} g \in Q_{q}$. Since $q<0<\operatorname{Re} c$ and $q<\operatorname{Re} a$, Theorem 5 (ii) shows that there is $\phi \in Q_{q}$ such that

$$
I^{c} x^{a} \phi(x)={ }^{\circ} x^{a} I^{c} \psi(x),={ }^{\circ} x^{a} g(x) .
$$

Since $x^{a} \phi(x) \in Q_{q-\alpha}$ where $\alpha=\operatorname{Re} a$, and $q-\alpha<0, x^{a} \phi(x) \in Q_{0}$. So

$$
x^{a} \phi(x)={ }^{\circ} I^{-c} x^{a} g(x) \text {, whence } x^{-a} I^{-c} x^{a} g(x)={ }^{\circ} \phi(x) \in Q_{q} .
$$

Thus Theorem 9 is applicable if $\operatorname{Re} b<\operatorname{Re} c$, and this completes the proof.

Lemma 5. If $\iota \theta$ is purely imaginary, $0<\operatorname{Re} k<\operatorname{Re} c$ and $I^{-c} f$ exists, then $I^{i \theta} f$, defined as $I^{-1} I^{1+}{ }^{1} \theta f$, is locally integrable on $[0, d)$ and

$$
\begin{aligned}
& I^{c+i \theta} I^{-c} f=I^{i \theta} f=I^{-c} I^{c+i \theta} f, \\
& I^{k+i \theta} I^{-k} f=I^{i \theta} f=I^{-k} I^{k+i \theta} f .
\end{aligned}
$$

By definition of $I^{-c} f$ there is locally integrable $\phi$ such that $f=I^{c} \phi$. By (3.2), that is,

$$
I^{1+i \theta} f=I^{1+i \theta} I^{c} \phi=I^{1+i \theta+c} \phi=I^{1} I^{c+i \theta} \phi,
$$

$$
I^{1+i \theta} f(x)={ }^{\circ} I^{1} I^{c+i \theta} \phi(x) .
$$

The left side is, by (3.1), the convolution of the locally integrable function $f(x)$ with the bounded function $x^{i \theta} / \Gamma(1+i \theta)$, and it is therefore continuous in $[0, d)$. The right side of (7.16) is an indefinite integral of the locally integrable function $I^{c+i \theta} \phi$; it is therefore locally absolutely continuous, and in particular continuous. Thus in (7.16) equality holds for all $x$ in $[0, d)$, not merely almost all.

Since the right side of (7.16) is locally absolutely continuous, so is the left, and differentiation gives

$$
I^{-1} I^{1+i \theta} f(x)={ }^{0} I^{c+i \theta} \phi(x) .
$$

Thus our definition of $I^{i \theta} f$ defines almost everywhere a locally integrable function, and also we obtain the first equation to be proved, namely

$$
I^{i \theta} f=I^{c+i \theta} I^{-c} f \text {. }
$$


By (3.2),

$$
I^{c} I^{c+i \theta} \phi=I^{2 c+i \theta} \phi=I^{c+i \theta} I^{c} \phi=I^{c+i \theta} f,
$$

and $I^{c+i \theta} \phi$ is locally integrable, whence

$$
I^{i \theta} f=I^{c+i \theta} \phi=I^{-c} I^{c+i \theta} f
$$

which is the second equation.

The other two equations will follow in the same way, with $c$ replaced by $k$, from existence of $I^{-k} f$. Using (3.2), $f=I^{c} \phi=I^{k} I^{c-k} \phi$ and $I^{c-k} \phi$ is locally integrable; consequently $I^{-k} f$ exists as required.

\section{Wider validity of the explicit solutions}

The next two theorems show that (7.7), which satisfies our integral equation under the conditions of Theorem 8 , including $\operatorname{Re} b>0$, also satisfies it under suitable conditions including $\operatorname{Re} b<0$; and similarly (7.12). then

Theorem 11. If $\operatorname{Re} b<0, \operatorname{Re} c>0, q<0, q<\operatorname{Re} a$, and $I^{b-c} g$ exists in $Q_{q}$,

$$
f(x)=x^{-a} I^{-b} x^{a} I^{b-c} g(x)
$$

is still in $Q_{q}$ and still satisfies (7.6) (compare Theorem 8). Further, its fractional derivative $I^{b} f$ exists and is in $Q_{q}$.

By Theorem 5 (i) with $b$ replaced by $-b$, there is $\phi \in Q_{q}$ such that

$$
I^{-b} x^{a}\left\{I^{b-c} g(x)\right\}={ }^{\circ} x^{a} I^{-b} \phi(x) .
$$

So (8.1) does define a function $f$, and $f=I^{-b} \phi$ where $\phi \in Q_{q}$. Thus $I^{b} f$ exists and is in $Q_{q}$, which proves the conclusion stated last.

Since $q<0<\operatorname{Re}(-b)$ and $\phi \in Q_{q},(2.12)$ with $a=0$ and $k=-b$ gives that $I^{-b} \phi \in Q_{q+b}$. And since $x^{-b}$ is locally bounded, it follows that

$$
x^{a} f(x)=x^{-b}, x^{q+b} I^{-b} \phi(x)
$$

is locally integrable, whence $f \in Q_{q}$.

Substituting (8.1) in (7.6), the left side becomes

$$
H(a, b, c) x^{-a} I^{-b} x^{a} I^{b-c} g(x) .
$$

Replacing $\lambda$ by $-b$ and $f$ by $I^{b-c} g$ in Theorem 3 , this expression is equal almost everywhere to $H(a, 0, c-b) I^{b-c} g=I^{c-b} I^{b-c} g=g$. So (7.6) is satisfied and the theorem is proved.

Theorem 12. If $\operatorname{Re} b>\operatorname{Re} c, \operatorname{Re} c>0, q<0, q<\operatorname{Re}(a+b-c)$ and $I^{-b} g$ exists in $Q_{q}$, then

$$
,(x)=x^{-b} I^{b-c} x^{c-a} I^{-b} x^{a+b-c} g(x),
$$

is still in $Q_{q}$ and still satisfies (7.11) (compare Theorem 9). Further, its fractional derivative $I^{c-b} f$ exists and is in $Q_{q}$.

This theorem can be proved using Theorem 11 in much the same way ag Theorem 9 is proved above using Theorem 8 . However a detour is needed to 
obtain the last conclusion by this method. A proof independent of Theorem 8 is given instead, since it gives greater reliability with little extra length.

By data there is $h \in Q_{q}$ such that $l^{b} h=g$.

By Theorem 5(ii) with $a$ replaced by $a+b-c$, there is $\phi \in Q_{q}$ such that

$$
I^{b} x^{a+b-c} \phi(x)={ }^{\circ} x^{a+b-c} I^{b} h(x) \text {. }
$$

By Theorem 5(i) with $a$ and $b$ replaced by $b$ and $b-c$ respectively, there is $\psi \in Q_{q}$ such that

$$
I^{b-c} x^{b} \phi(x)={ }^{o} x^{b} I^{b-c} \psi(x) \text {. }
$$

Thus $I^{b-c} \psi(x)={ }^{\circ} x^{-b} I^{b-c} x^{b} \phi(x)=x^{-b} I^{b-c} x^{c-a} I^{-b} x^{a+b-c} g(x)$; so (8.2) does define a function $f$, and $f=I^{b-c} \psi$ where $\psi \in Q_{q}$. Hence $I^{c-b} f$ exists and is in $Q_{q}$, as stated.

Since $q<0$ we have, writing $\operatorname{Re} b=\beta$ and $\operatorname{Re} c=\gamma$ as usual,

$$
x^{q}\left|I^{b-c} \psi(x)\right| \leqq \int_{0}^{x}\left|\frac{(x-t)^{b-c-1}}{\Gamma(b-c)}\right| x^{q}|\psi(t)| d t \leqq \int_{0}^{x} \frac{(x-t)^{\beta-y-1}}{|\Gamma(b-c)|} t^{q}|\psi(t)| d t
$$

As $x^{q}|\psi(x)|$ is locally integrable this shows that $x^{q} f(x)$ is also, that is, $f \in Q_{q}$.

Finally, we substitute (8.2) in (7.11). Using [(10), 2.9(2)] and the notation (7.14), the left side of (7.11) becomes

$$
\begin{aligned}
& \int_{0}^{x} \frac{(x-s)^{c-1}}{\Gamma(c)} \cdot F\left(a^{\prime}, b^{\prime},{ }^{m}, c^{\prime} 1-\frac{x}{s}\right)\left(\frac{x}{s}\right)^{c-a-b} f(s) d s \\
& =x^{c-a-b} H\left(a^{\prime}, b^{\prime}, c\right) x^{-a^{\prime}} I^{-b^{\prime}} x^{a^{\prime}} I^{-b} g^{\prime}(x) \\
& ={ }^{\circ} x^{c-a-b} H\left(a^{\prime}, 0, c-b^{\prime}\right) I^{-b} g^{\prime}(x) \text {. }
\end{aligned}
$$

Here we have used Theorem 3 with $\lambda=-b^{\prime}=b-c$, with the letters $q, a, b$ accented, and with $f$ replaced by $I^{-b} g^{\prime}$. The correctness of this is easily enough verified; in particular, since the right side of $(8.3)$ is $g^{\prime}(x)$,

$$
x^{q^{\prime}} I^{-b} g^{\prime}(x)={ }^{\circ} x^{q^{\prime}} x^{a+b-c} \phi(x)=x^{q+i \theta} \phi(x)
$$

and this is locally integrable since $\phi \in Q_{q}$ and $i \theta=a+b-c+\gamma-\alpha-\beta$ is purely imaginary. Now (8.4) is simply

$$
x^{c-a-b} I^{b} I^{-b} g^{\prime}(x)=x^{c-a-b} g^{\prime}(x)=g(x)
$$

so that (8.2) does indeed satisfy (7.11).

\section{Alternative necessary conditions and sufficient conditions}

Although Theorem 8 is approximately converse to Theorem 7, its companion Theorem 9 lacks this character because the differentiability required of $g$ refers to $I^{-c} x^{a} g(x)$ rather than to $I^{-c} g(x)$. However in Theorem 10 we were able to switch from one of these hypotheses to the other by means of Theorem 5, thus making Theorem 10 approximately converse to Theorem 7 .

It is possible to write Theorem 9 also in terms of $I^{-c} g$, at the cost of an extra assumption $q<0$ (see Theorem 9, Extension (iii) below). But this is only one of several alternative versions of that theorem. In this section we use Theorems 
5 and 6 to obtain similarly a variety of different versions of Theorems 7,8 and 9. In some cases the different "versions" are corollaries, or actually equivalent statements; in others they are extensions, particularly those with $q=0$.

Theorem 5 Corollary. Suppose $q<0<\operatorname{Re} b$ and $q<\operatorname{Re} a$. If $x^{-a} I^{-b} x^{a} g(x)$ exists in $Q_{q}$ then $I^{-b} g$ exists in $Q_{q}$, and conversely.

(i) Suppose $\phi(x)=x^{-a} I^{-b} x^{a} g(x) \in Q_{q}$. Then $I^{b} x^{a} \phi(x)={ }^{\circ} x^{a} g(x) . \quad$ Вy Theorem 5(i) there is $\psi \in Q_{q}$ such that $I^{b} x^{a} \phi(x)={ }^{\circ} x^{a} I^{b} \psi(x)$. So $g(x)={ }^{\circ} I^{b} \psi(x)$; and $\psi$ is locally integrable since $Q_{q} \subset Q_{0}$. Thus $I^{-b} g$ exists and is in $Q_{q}$.

(ii) Suppose $\psi=I^{-b} g \in Q_{q}$. By Theorem 5(ii) there is $\phi \in Q_{q}$ such that $I^{b} x^{a} \phi(x)={ }^{\circ} x^{a} I^{b} \psi(x),={ }^{\circ} x^{a} g(x)$. Since $x^{a} \phi(x) \in Q_{q-\alpha}$ where $\alpha=\operatorname{Re} a$, and since $q-\alpha<0$ implies that $Q_{q-\alpha} \subset Q_{0}, x^{a} \phi(x)$ is locally integrable. Thus $x^{a} \phi(x)={ }^{\circ} I^{-b} x^{a} g(x)$, giving the conclusion.

The above proofs can be turned round to show that Theorem 5 is a consequence of Theorem 5 Corollary, so that the latter is equivalent to the former. Similarly Theorem 6 is equivalent to Theorem 6 Corollary below. Before introducing this corollary we consider a lemma.

Lemma 6. If $q<0$ and $g \in Q_{q}$ then $\bar{g} \in Q_{q}$. Here $\bar{g}$ is the function whose values are given by

$$
\bar{g}(x)=\frac{1}{x} \int_{0}^{x} g(t) d t
$$

Supposing $0<l<d$, so that $x^{q} g(x)$ is integrable on $(0, l)$,

$$
\begin{aligned}
\int_{0}^{t} x^{q^{i}}|\bar{g}(x)| d x & \leqq \int_{0}^{l} x^{q-1} d x \int_{0}^{x}|g(t)| d t=\int_{0}^{l}|g(t)| d t \int_{t}^{t} x^{q-1} d x \\
& =\int_{0}^{l}|g(t)| \frac{t^{q}-l^{q}}{-q} d t \leqq-\frac{1}{q} \int_{0}^{l} t^{q}|g(t)| d t .
\end{aligned}
$$

The last integral is finite, and this proves the lemma.

Lemma 6 becomes false if $q=0$, as we have already noted at (5.13).

Theorem 6 Corollary. Suppose $\operatorname{Re} a>0$ and $\operatorname{Re} b>0$. If $x^{-a} I^{-b} x^{a} g(x)$ exists in $Q_{0}$ then $I^{-b} g$ and $\overline{I^{-b} g}$ exist in $Q_{0}$, and conversely.

Proof of this statement proceeds almost word for word the same as the proof of Theorem 5 Corollary, so it is omitted. The two corollaries can be combined, using Lemma 6, in the form: Suppose $q \leqq 0<\operatorname{Re} b$ and $q<\operatorname{Re} a$. If

$$
x^{-a} I^{-b} x^{a} g(x)
$$

exists in $Q_{q}$ then $I^{-b} g$ and $\overline{I^{-b} g}$ exist in $Q_{q}$, and conversely.

The extensions of Theorems 7, 8 and 9, with the original statements, now f ollow. $\dagger$

$\dagger$ It will be appropriate in subsequent reference to regard Theorem 4 also as one of this series and to refer to it as Theorem $4 A$. 
Theorem 7A. If $\operatorname{Re} c>0, q \leqq 0, q<\min (\operatorname{Re} a, \operatorname{Re} b), f$ is in $Q_{q}$ and

$$
\int_{0}^{x} \frac{(x-t)^{c-1}}{\Gamma(c)} F\left(a, b, c, 1-\frac{x}{t}\right) f(t) d t={ }^{\circ} g(x),
$$

then $I^{-c} g$ exists in $Q_{q}$.

Further $\overline{I^{-c} g}, x^{-a} I^{-c} x^{a} g(x)$ and $x^{-b} I^{-c} x^{b} g(x)$ also exist in $Q_{q}$.

If $q<0$ it is immediate from Lemma 6 that $\overline{I^{-c} g} \in Q_{q}$. And Theorem 5 Corollary with $b$ replaced by $c$ shows that $x^{-a} I^{-c} x^{a} g(x)$ exists in $Q_{q}$. From this it follows that $x^{-b} I^{-c} x^{b} g(x)$ exists in $Q_{q}$, since the hypothesis is symmetrical in $a$ and $b$.

If $q=0$ we have from (6.4) that

$$
\overline{I^{-c} g}(x)=f(x)-\frac{a b}{x} \int_{0}^{x} d s \int_{0}^{s} F\left(a+1, b+1,2,1-\frac{s}{t}\right) \frac{f(t)}{t} d t .
$$

The argument used in Theorem 6(i), second paragraph, applies to $\overline{I^{-c} g}$, and proves that this function is in $Q_{0}$. That the other two functions exist and are in $Q_{0}$ then follows as in the case $q<0$, but using Theorem 6 Corollary.

Remark. Theorem 7A thus establishes three more necessary conditions for existence of a solution of (9.2). Theorem 9 has already shown the partial sufficiency of one of these; and other corollaries below provide similar results relating to the others.

Theorem 8A. If $\operatorname{Re} c>0, \operatorname{Re} b>0, q<\operatorname{Re} a, q<0$ and $I^{-c} g$ exists in $Q_{q}$, then equation (9.2) has a solution $f$ in $Q_{q}$, given by (7.7).

This conclusion still holds if the hypothesis " $q<0$ and $I^{-c} g$ exists in $Q_{q}$ " is replaced by any of the following three:

(i) $q \leqq 0$ and $x^{-a} I^{-c} x^{a} g(x)$ exists in $Q_{q}$;

(ii) $q \leqq 0$ and $x^{-b} I^{-c} x^{b} g(x)$ exists in $Q_{q}$;

(iii) $q=0$ and $\overline{I^{-c} g}$ exists in $Q_{0}$.

Supposing $q<0$, (i) follows from Theorem 5 Corollary with $b$ replaced by $c$, and (ii) from Theorem 5 Corollary with $a$ and $b$ replaced by $b$ and $c$.

If $q=0$, (iii) is obtained by the same proof as Theorem 8 itself, but using Theorem 6(ii) at (7.9) instead of Theorem 5(ii). This use of Theorem 6(ii) requires that $I^{-c} g$ should exist in $Q_{0}$, a datum which appears now to have been dropped; but by definition (9.1) it is implicit in (iii) that $I^{-c} g(x)$ should exist almost everywhere and be locally integrable on $[0, d)$, that is, that $I^{-c} g$ should exist in $Q_{0}$.

If $q=0$, (i) implies (iii) by Theorem 6 Corollary with $b$ replaced by $c$, and (ii) implies (iii) by Theorem 6 Corollary with $a$ and $b$ replaced by $b$ and $c$. This proves (i) and (ii) in the case $q=0$.

Theorem 9A. If $\operatorname{Re} c>0, \operatorname{Re} b<\operatorname{Re} c, q<\min (\operatorname{Re}(a+b-c), \operatorname{Re} b)$ and $x^{-a} I^{-c} x^{a} g(x)$ exists in $Q_{q}$, then equation (9.2) has a solution $f$ in $Q_{q}$, given by (7.12). 
This conclusion still holds if the hypothesis " $x^{-a} I^{-c} x^{a} g(x)$ exists in $Q_{q}$ " is replaced by any of the following four:

(i) $x^{c-a-b} I^{-c} x^{a+b-c} g(x)$ exists in $Q_{q}$;

(ii) $q \leqq 0$ and $x^{-b} I^{-c} x^{b} g(x)$ exists in $Q_{q}$;

(iii) $q<0$ and $I^{-c} g$ exists in $Q_{q}$;

(iv) $q=0$ and $\overline{I^{-c} g}$ exists in $Q_{0}$.

Hypothesis (i) says, using (7.14), that $I^{-c} g^{\prime}$ exists in $Q_{q^{\prime}}$. Substituting this statement for the second paragraph of the proof of Theorem 9 we obtain a proof when (i) holds.

Since $\operatorname{Re}(b-c)<0$ the data imply that $q<\operatorname{Re} a$. Theorem 5 Corollary with $b$ replaced by $c$ shows that (iii) implies the original hypothesis. Again, Theorem 5 Corollary with $a$ and $b$ replaced by $b$ and $c$ shows that (ii) implies (iii) provided $q<0$. Thus (iii), and (ii) with $q<0$, each imply the original hypothesis and so the conclusion.

If (iv) holds then so does the original hypothesis, by Theorem 6 Corollary with $b$ replaced by $c$. And if (ii) holds with $q=0$ then so does (iv), by Theorem 6 Corollary with $a$ and $b$ replaced by $b$ and $c$. Thus (iv), and (ii) with $q=0$, each imply the original hypothesis and so the conclusion.

\section{Another hypergeometric integral equation}

We now deduce theorems on solutions of the integral equation (1.5), namely

$$
\int_{0}^{x} \frac{(x-t)^{c-1}}{\Gamma(c)} F\left(a, b, c, 1-\frac{t}{x}\right) f(t) d t={ }^{\circ} g(x) \text { for } 0<x<d,
$$

where $0<d \leqq \infty$. This equation differs from that discussed in most of the rest of this paper only in the fourth variable of the hypergeometric function, which is now $1-t / x$ instead of $1-x / t$. We convert (10.1) into the form of (1.4) (that is, (9.2)) by substitutions, and then apply Theorems $4,7 \mathrm{~A}$ and $8 \mathrm{~A}$ to obtain corresponding theorems about (10.1).

This conversion may be achieved using either of Kummer's relations [(10), 2.9(3) and (4)]. Using the former, (10.1) is equivalent to

$$
\int_{0}^{x} \frac{(x-t)^{c-1}}{\Gamma(c)}-F\left(a, b^{\prime}, c, 1-\frac{x}{t}\right) f^{\prime}(t) d t={ }^{\circ} g^{\prime}(x) \text { for } 0<x<d,
$$

where

and also

$$
\left.\begin{array}{rl}
b^{\prime}=c-b, \quad f^{\prime}(t) & =t^{-a} f(t), \quad g^{\prime}(x)=x^{-a} g(x), \\
q^{\prime} & =q+\operatorname{Re} a .
\end{array}\right\}
$$

Theorem 4B. If $q \leqq \min (\operatorname{Re}(c-a), \operatorname{Re}(c-b)), q<\min (0, \operatorname{Re}(c-a-b))$, $\operatorname{Re} c>0, f$ is in $Q_{q}$ and (10.1) holds with $g(x)={ }^{\circ} 0$, then $f(x)={ }^{\circ} 0$.

Equation (10.2) holds with $g^{\prime}(x)={ }^{\circ} 0$, by (10.3), and we propose to apply Theorem 4 to this equation. Using (10.3) our data give

$$
q^{\prime} \leqq \min \left(\operatorname{Re} c, \operatorname{Re}\left(a+b^{\prime}\right)\right), \quad q^{\prime}<\min \left(\operatorname{Re} a, \operatorname{Re} b^{\prime}\right) ;
$$


and $x^{a^{\prime}} f^{\prime}(x)$ is locally integrable because $\left|x^{a^{\prime}} f^{\prime}(x)\right|=\left|x^{q+a} \cdot x^{-a} f(x)\right|=\left|x^{a} f(x)\right|$. Thus Theorem 4 shows that $f^{\prime}(x)={ }^{\circ} 0$, and so $f(x)={ }^{\circ} 0$ as required.

Theorem 7B. If $\operatorname{Re} c>0, q \leqq-\operatorname{Re} a, q<\min (0, \operatorname{Re}(c-a-b)), f$ is in $Q_{q}$ and (10.1) holds, then $I^{-c} g$ exists in $Q_{q}$.

Further $x^{a} I^{-c} x^{-a} g(x), x^{a+b-c} I^{-c} x^{c-a-b} g(x)$ and $x^{a} I^{-c} x^{-a} g(x)$ exist in $Q_{q}$.

Using (10.3) our data give

$$
\operatorname{Re} c>0, \quad q^{\prime} \leqq 0, \quad q^{\prime}<\min \left(\operatorname{Re} a, \operatorname{Re} b^{\prime}\right) ;
$$

also $f^{\prime} \in Q_{q}$, as in the preceding proof. So Theorem 7A applies to (10.2), giving that $I^{-c} g^{\prime}$ and its average, $x^{-a} I^{-c} x^{a} g^{\prime}(x)$ and $x^{-b^{\prime}} I^{-c} x^{b^{\prime}} g^{\prime}(x)$ all exist in $Q_{q^{\prime}}$. These conclusions give, in order, that

$$
x^{a} I^{-c} x^{-a} g(x), x^{a} \overline{I^{-c} x^{-a} g(x)}, I^{-c} g(x), x^{a+b-c} I^{-c} x^{c-a-b} g(x)
$$

all exist in $Q_{q}$, as required.

Theorem 8B. If $\operatorname{Re} c>0, \operatorname{Re} b<\operatorname{Re} c, q<0, q \leqq-\operatorname{Re} a$ and $I^{-c} g$ exists in $Q_{q}$, then (10.1) has a solution $f$ in $Q_{q}$, expressible by

$$
f(x)=I^{b-c} x^{a} I^{-b} x^{-a} g(x) .
$$

The assumption that $I^{-c} g$ exists in $Q_{q}$ may be replaced by either of:

(i) $x^{a+b-c} I^{-c} x^{c-a-b} g(x)$ exists in $Q_{q}$;

(ii) $x^{a} I^{-c} x^{-a} g(x)$ exists in $Q_{q}$, supplemented by $x^{a} \overline{I^{-c} x^{-a} g(x)}$ also existing in $Q_{q}$ if $q=-\operatorname{Re} a$.

Using (10.3) our data show that $\operatorname{Re} c>0, \operatorname{Re} b^{\prime}>0, q^{\prime}<\operatorname{Re} a$ and $q^{\prime} \leqq 0$. Also we are supposing that $x^{-a} I^{-c} x^{a} g^{\prime}(x)$ exists in $Q_{q^{\prime}}$; or alternatively that $x^{-b^{\prime}} I^{-c} x^{b^{\prime}} g^{\prime}(x)$ exists in $Q_{q^{\prime}}$; or again that $I^{-c} g^{\prime}$ exists in $Q_{q^{\prime}}$, supplemented if $q^{\prime}=0$ by its average being also in $Q_{0}$. Under these conditions Theorem $8 \mathrm{~A}$ applies to (10.2), showing that it has a solution $f^{\prime}$ in $Q_{q^{\prime}}$, expressible by

$$
f^{\prime}(x)=x^{-a} I^{-b^{\prime}} x^{a} I^{b^{\prime}-c} g^{\prime}(x) .
$$

Thus (10.1) has a solution $f(x)=x^{a} f^{\prime}(x)$ expressible by (10.4) after using (10.3); and $f \in Q_{q}$.

Theorem 9B. If $\operatorname{Re} c>0, \operatorname{Re} b>0, q<\operatorname{Re}(c-a-b), q \leqq-\operatorname{Re} b$ and $I^{-c} g$ exists in $Q_{q}$, then (10.1) has a solution $f$ in $Q_{q}$, expressible by

$$
f(x)=x^{a+b-c} I^{-b} x^{c-a} I^{b-c} x^{-b} g(x) .
$$

The assumption that $I^{-c} g$ exists in $Q_{q}$ may be replaced by either of:

(i) $x^{a+b-c} I^{-c} x^{c-a-b} g(x)$ exists in $Q_{q}$;

(ii) $x^{b} I^{-c} x^{-b} g(x)$ exists in $Q_{q}$, supplemented by $x^{b} \overline{I^{-c} x^{-b} g(x)}$ also existing in $Q_{q}$ if $q=-\operatorname{Re} b$.

Instead of [(10), 2.9(3)] and Theorem 9A, we use [(10), 2.9(4)] and Theorem 
$8 \mathrm{~A}$ to establish this theorem. By [(10), 2.9(4)], equation (10.1) is equivalent to

$$
\int_{0}^{x} \frac{(x-t)^{c-1}}{\Gamma(c)} F\left(a^{\prime}, b, c, 1-\frac{x}{t}\right) f^{\prime}(t) d t={ }^{\circ} g^{\prime}(x)
$$

where

and also

$$
\left.\begin{array}{c}
a^{\prime}=c-a, \quad f^{\prime}(t)=t^{-b} f(t), \quad g^{\prime}(x)=x^{-b} g(x), \\
q^{\prime}=q+\operatorname{Re} b .
\end{array}\right\}
$$

Using (10.7) our data show that $\operatorname{Re} c>0, \operatorname{Re} b>0, q^{\prime}<\operatorname{Re} a^{\prime}$ and $q^{\prime} \leqq 0$. Also we are supposing that $x^{-b} I^{-c} x^{b} g^{\prime}(x)$ exists in $Q_{q^{\prime}}$; or alternatively that $x^{-a^{\prime}} I^{-c} x^{a^{\prime}} g^{\prime}(x)$ exists in $Q_{q^{\prime}}$; or again that $I^{-c} g^{\prime}$ exists in $Q_{q^{\prime}}$, supplemented if $q^{\prime}=0$ by its average being also in $Q_{0}$. Thus Theorem 8A applies to (10.6), showing that it has a solution $f^{\prime}$ in $Q_{q^{\prime}}$, expressible by

$$
f^{\prime}(x)=x^{-a^{\prime}} I^{-b} x^{a^{\prime}} I^{b-c} g^{\prime}(x) .
$$

Thus (10.1) has a solution $f(x)=x^{b} f^{\prime}(x)$ expressible by (10.5) after using (10.7); and $f \in Q_{q}$.

Remark. Comparing the explicit solutions (10.4) and (10.5) with (7.7) and (7.12), we see that formal reversal of the order of operations in the solution formulae for (1.4) gives the solution formulae for (1.5).

Theorem 10B. If $q<\min (0, \operatorname{Re}(c-a-b)), q \leqq \min (-\operatorname{Re} a,-\operatorname{Re} b)$ $\operatorname{Re} c>0$, and $I^{-c} g$ exists in $Q_{q}$, then the equation

$$
\int_{0}^{x} \frac{(x-t)^{c-1}}{\Gamma(c)} F\left(a, b, c, 1-\frac{t}{x}\right) f(t) d t={ }^{\circ} g(x)
$$

has a unique solution $f$ in $Q_{q}$, given by (10.4) if $\operatorname{Re} b<\operatorname{Re} c$ and by (10.5) if $\operatorname{Re} b>0$.

The uniqueness follows from Theorem $4 \mathrm{~B}$; the rest from Theorem $8 \mathrm{~B}$ when $\operatorname{Re} b<\operatorname{Re} c$ and from Theorem 9B when $\operatorname{Re} b>0$.

Remark. Theorem 10 is slightly less complete than Theorem 10B, in that equality is not permitted in either inequality restricting $q$. It can be shown that $q=\min (0, \operatorname{Re}(a+b-c))$ cannot be permitted in Theorem 10 , by the example

$$
\begin{gathered}
q=0, \quad b=c=1, \quad \operatorname{Re} a>0, \\
g(x)=1 / \log (3 / x) \text { if } 0<x<1, \quad g(x)=1 / \log 3 \text { if } x \geqq 1 .
\end{gathered}
$$

Elementary calculations show that the only solution of the integral equation of Theorem 10 is then not in $Q_{0}$, because for $0<x<1$ it is

$$
f(x)=\frac{a}{x \log (3 / x)}+\frac{1}{x\{\log (3 / x)\}^{2}}
$$

It remains to be seen whether $q=\min (\operatorname{Re} a, \operatorname{Re} b)$ can be permitted in Theorem 10. 


\section{REFERENCES}

(1) TA Li, A new class of integral transforms, Proc. Amer. Math. Soc. 11 (1960), 290-298.

(2) R. G. Buschman, An inversion integral, Proc. Amer. Math. Soc. 13 (1962), 675-677.

(3) T. P. Higgins, An inversion integral for a Gegenbauer transformation, J. Soc. Indust. Appl. Math. 11 (1963), 886-893.

(4) K. N. SRIVastava, A class of integral equations involving ultraspherical polynomials as kernel, Proc. Amer. Math. Soc. 14 (1963), 932-940.

(5) A. ERDÉLYI, An integral equation involving Legendre functions, J. Soc. Indust. Appl. Math. 12 (1964), 15-30.

(6) T. P. Higgins, A hypergeometric function transform, J. Soc. Indust. Appl. Math. 12 (1964), 601-612.

(7) JET WIMP, Two integral transform pairs involving hypergeometric functions, Proc. Glasgow Math. Assoc. 7 (1965), $42-44$.

(8) K. N. SRIVAStava, Fractional integration and integral equations with polynomial kernels, J. London Math. Soc. 40 (1965), 435-440.

(9) H. KoBer, On fractional integrals and derivatives, Quart. J. Math. (Oxford) 11 (1940), 193-211 (Thm. 2(3.4) (i) with $p=1$ ).

(10) A. ERDÉLYI et al., Higher Transcendental Functions, vol. 1 (McGraw Hill, 1953).

UNIVERSITY OF EDINBURGH and

UNIVERSITY OF MELBOURNE 